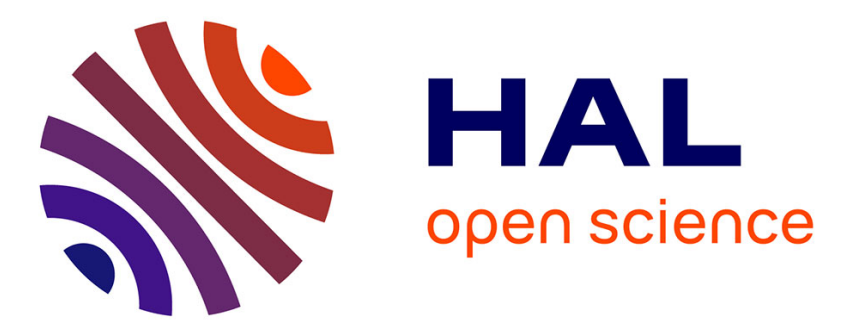

\title{
A Model-Point Approach to Indifference Pricing of Life Insurance Portfolios with Dependent Lives
}

\author{
Christophette Blanchet-Scalliet, Diana Dorobantu, Yahia Salhi
}

\section{To cite this version:}

Christophette Blanchet-Scalliet, Diana Dorobantu, Yahia Salhi. A Model-Point Approach to Indifference Pricing of Life Insurance Portfolios with Dependent Lives. Methodology and Computing in Applied Probability, 2019, 21 (423-448), 10.1007/s11009-017-9611-2 . hal-01258645

\section{HAL Id: hal-01258645 \\ https://hal.science/hal-01258645}

Submitted on 19 Jan 2016

HAL is a multi-disciplinary open access archive for the deposit and dissemination of scientific research documents, whether they are published or not. The documents may come from teaching and research institutions in France or abroad, or from public or private research centers.
L'archive ouverte pluridisciplinaire HAL, est destinée au dépôt et à la diffusion de documents scientifiques de niveau recherche, publiés ou non, émanant des établissements d'enseignement et de recherche français ou étrangers, des laboratoires publics ou privés. 


\title{
A Model-Point Approach to Indifference Pricing of Life Insurance Portfolios with Dependent Lives*
}

\author{
$\begin{array}{lll}\text { C. BLANCHET-SCALLIET } & \text { D. DOROBANTU } & \text { Y. SALHI } \\ & \end{array}$
}

January 14, 2016

\begin{abstract}
In this paper, we study the pricing of life insurance portfolios in the presence of dependent lives. We assume that an insurer with an initial exposure to $n$ mortality-contingent contracts wanted to acquire a second portfolio constituted of $m$ individuals. The policyholders' lifetimes in these portfolios are correlated with a Farlie-Gumbel-Morgenstern (FGM) copula, which induces a dependency between the two portfolios. In this setting, we compute the indifference price charged by the insurer endowed with an exponential utility. The optimal price is characterized as a solution to a backward differential equation (BSDE). The latter can be decomposed into $(n-1) n$ ! auxiliary BSDEs. In this general case, the derivation of the indifference price is computationally infeasible. Therefore, while focusing on the example of death benefit contracts, we develop a model point based approach in order to ease the computation of the price. It consists on replacing each portfolio with a single policyholder that replicates some risk metrics of interest. Also, the two representative agents should adequately reproduce the observed dependency between the initial portfolios.
\end{abstract}

Keywords: indifference pricing, representative contract, utility maximization, life insurance.

JEL classification: G11; G13; G22;

\section{Introduction}

Life insurance risks, especially mortality and longevity, have gained importance not only among practitioners but also from an academic point of view. Many insurance companies are exposed to these risks and are continuously invited by regulators to manage them accurately. Various

*This work benefited from the financial support of the ANR project "LoLitA" (ANR-13-BS01-0011) and the BNP Paribas Cardif Chair "Data Analytics \& Models for Insurance". The views expressed in this document are the authors owns and do not necessarily reflect those endorsed by BNP Paribas Cardif.

${ }^{\dagger}$ Ecole Centrale de Lyon, Institut Camille Jordan Université de Lyon CNRS UMR 5208, France. Contact: christophette.blanchet@ec-lyon.fr

${ }^{\ddagger}$ ISFA, Institut Camille Jordan Université de Lyon CNRS UMR 5208, France. Contact: diana.dorobantu@ univ-lyon1.fr

§ISFA, Laboratoire SAF, Universiteé de Lyon, Université Lyon 1, France. Contact: yahia.salhi@ univ-lyon1.fr 
strategies have been developed trying to fully or partially hedge out such risks Barrieu et al. (2012). Among these strategies, the natural hedge between the mortality component and the longevity risk component arises as a key element of insurers mitigation techniques Cox and Lin (2007).

Aside from the hedging problem of such risks, the pricing is also of paramount importance. In fact, the insurance market is inherently incomplete to the extent that no unique pricing probability measure is available. Thus, various pricing procedures have been proposed in the literature: using indifference pricing as in Ludkovski and Young (2008), the Sharpe ration as in Bayraktar et al. (2009) or characterizing the price via the good deal bounds in Delong (2012). In Ludkovski and Young (2008), an indifference pricing procedure is developed using a fully stochastic framework that extends the original pricing approach introduced by Hodges and Neuberger (1989) to mortality-contingent claims.

In this paper, we consider the indifference pricing of mortality contingent claims when the utility is exponential. Unlike Ludkovski and Young (2008), we consider an insurer with initial exposure to such a risk, holding a portfolio of $n$ policies. The latter intended to sell $m$ more contracts to individuals that are correlated to the initial exposure. The dependence between individuals' lifetimes and thus portfolios is due, for example, to medical breakthroughs or environmental factors that affect a whole population. Also, portfolios of different age structures may be dependent or even negatively dependent. Indeed, as noted by Marceau and Gaillardetz (1999), Milevsky and Promislow (2001) and Cairns et al. (2004) among others, insurers may be intended to mix different life insurance contracts and portfolios in order to naturally hedge the underlying risk. The authors explore impacts of mortality changes on life insurance and annuities separately, and investigates a simple combination of life and pure endowment life contracts.

To take into account this dependency we consider the density framework introduced in El Karoui et al. (2013) that relates individuals' death times in such a way that the death of one impacts the intensity of the remaining individuals. The density approach, considered in this paper, provides a new vision for modeling the dependency of deaths among a population not only from a theoretical point of view but also for practical use. According to El Karoui et al. (2013), this amounts to saying that one individual's intensity will have a jump when the another individual is deceased. More precisely, the dependence effect arises due to the fact that the occurrence of first death helps localize the other death occurrences in the support of its conditional law. The exponential indifference price and the hedging strategy for the guaranteed payments process is derived from the solution to the utility optimization problem. We lay the emphasis on the dependency between the policyholders and thus consider a general portfolio not only with heterogeneous lives, i.e. dependent policyholders whose lives are not necessarily governed by the same hazard rate. By doing so, we implicitly consider that the underlying individuals are not homogeneous with regard to their health status or other relevant risk characteristics. We further assume that the portfolio itself lacks of homogeneity in the sense that the policyholders may attain different term insurance contracts sharing the same duration.

In Section 1, we formulate the problem of indifference pricing of an insurer with constant relative risk aversion. In order to derive the price for selling $m$ additional contracts, we first focus on the value function of the insurer when she does not sell the $m$ contracts and the value function when she does. The first optimization problem is well studied in the literature for 
$n=1$, we here give a slight generalization following the same reasoning as in Kharroubi and Lim (2014). In the presence of the $m$ contracts we also use the same reasoning considered in Kharroubi and Lim (2014) to characterize the optimal value function of the optimization problem in terms of a solution of backward stochastic differential solutions (BSDE). The different BSDEs involved are given in Section 2. Then, the indifference price is solely determined by the solution of these BSDEs.

For the sake of applicability, we should note that the derivation of the indifference price when $n>1$ and $m>1$ is not feasible. Indeed since the death times are not ordered, to compute the value functions, we need to solve $(n-1) n$ ! BSDEs. So, it is difficult to use these results in numerical analyzes. Similar problems have been encountered by Ludkovski and Young (2008). Therefore, to overcome this numerical issue we consider that the insurer rely on a model point which consists on the construction of a representative policy for each portfolio. This policy is determined in such a way that the statistical characteristics as well as the risk profile of the aggregate exposure is replicated. By doing so, we consider two representative agents holding a contract that matches the $n$ (resp. $m$ ) policyholders' risk and an expected payment being close the initial one. The two model points also replicate the correlation between the initial portfolios. The correlation structure is parametrized using an FGM copula. In Subsection 2.1 we give the main characteristics of these agents: mortality intensities, guaranteed amounts and the correlation through the parameter of the FGM copula.

The remainder is organized as follows. In Section 1 we formulate the indifference pricing problem in the general setting of an insurer holding an exposure on a portfolio of $n$ policyholders wanting to sell $m$ additional contracts. Before, we introduce the financial and insurance markets. The latter is characterized by some policies whose payments are contingent on the death of the individual. In the presence of multiple lives we consider a particular type of dependency through the use of a copula and characterize the joint law as well as the intensity dynamic. Hence, in Section 2, we focus on the solution of the optimization problem using the BSDE tools. We also introduce the representative agents replicating the main risk characteristics of the initial exposures as well as the sold portfolio. In Section 3, the efficiency of the aggregation method is illustrated on synthetic portfolios with a given age structure, and the sensitivity of the construction methodology to the main portfolio parametrization is investigated. We also give the indifference price for death benefit contracts and analyze the impact of the size of

the considered portfolio, the mortality profile, the dependence structure and other relevant financial risk factors.

\section{Financial market, insurance contacts and mathematical set- ting}

In this section, we introduce the mathematical framework used to represent the financial market as well as the insurance contracts considered throughout the sequel. Some useful and classical assumptions are also introduced.

1.1 Financial model. Let $(\Omega, \mathcal{G}, \mathbb{P})$ be a complete probability space equipped with a standard (one-dimensional) Brownian motion $W=\left(W_{t}\right)_{t \geq 0}$. We denote by $\mathbb{F}:=\left(\mathcal{F}_{t}\right)_{t \geq 0}$ the right 
continuous complete filtration generated by $W$.

We consider a financial market on the time interval $[0, T]$ where $T>0$ corresponds to the term of the insurance contracts. The financial market consists of a riskless money market account with a constant interest rate $r$ and a reference portfolio of risky assets. The price processes $\hat{S}^{0}=\left(\hat{S}_{t}^{0}\right)_{t \in[0, T]}$ of the riskless bond and $\hat{S}=\left(\hat{S}_{t}\right)_{t \in[0, T]}$ of a share of the underlying risky portfolio are assumed to be solutions of the linear stochastic differential equations

$$
\begin{aligned}
d \hat{S}_{t}^{0} & =r \hat{S}_{t}^{0} d t, \quad \hat{S}_{0}^{0}=1, \\
d \hat{S}_{t} & =\hat{S}_{t}\left(\mu_{t} d t+\sigma_{t} d W_{t}\right), \quad \hat{S}_{0}=s_{0} \geq 0
\end{aligned}
$$

for $t \in[0, T]$, where $\mu$ and $\sigma$ are $\mathbb{F}$-predictable processes satisfying the classical assumptions to avoid arbitrage opportunities.

\section{Assumption 1.}

(i) The processes $\mu$ and $\sigma$ are bounded.

(ii) The process $\sigma$ is lower bounded by a positive constant $\underline{\sigma}$.

1.2 Insurance contracts. We consider term life insurance contracts, which provide coverage to policyholders for a limited period of time $T$. Two common examples are a pure endowment that pays an amount $c$ at time $T$ if the individual is alive at that time, and a temporary life annuity that pays $c$ per unit of time as long as the individual is alive, but at most until $T$. Another example for such contacts is death benefits contracts for which a beneficiary receives the guaranteed amount if the insured dies during the term of the contract, i.e. before $T$.

We assume that an insurance company issued $n$ contracts with different sums at risk $c_{i}$. Each individual $i \in\{1, \cdots, n\}$ pays an upfront price and has a guaranteed amount $c_{i}$. All the contracts have a maturity $T$. Such an exposure is contingent on the death of the individuals. Thus, we consider a family of random times $\boldsymbol{\tau}=\left(\tau_{1}, \cdots, \tau_{n}\right)$ taking values on $\mathbb{R}_{+}^{n}$ to represent the death times. In other words, each policyholder $i$ has a remaining lifetime $\tau_{i}$. Moreover, the insurer exposure on each individual denotes $F^{i}$. Here, in the case of term life insurance the guaranteed amount is $F_{\tau_{i} \wedge T}^{i}=c_{i} \mathbf{1}_{\left\{\tau_{i}<T\right\}}$.

In our setting, we consider that an individual's death has no impact on the financial market. Therefore, the vector $\tau$ is assumed to be independent of $\mathbb{F}$. However, the insurer's investment strategies can depend both on the market evolution and on the policyholders' remaining lifetimes. Therefore, we introduce the following filtrations $\mathbb{G}^{i}:=\left(\mathcal{G}_{t}^{i}\right)_{t \geq 0}, i \leq n$ is defined by

$$
\mathbb{G}^{i}:=\mathbb{F} \vee \mathbb{D}^{1} \vee \cdots \vee \mathbb{D}^{i}
$$

for any $t \geq 0$, where $\mathbb{D}^{k}=\left(\mathcal{D}_{t}^{k}\right)_{t \geq 0}, \mathcal{D}_{t}^{k}=\bigcap_{s>t} \tilde{\mathcal{D}}_{s}^{k}$ and $\tilde{\mathcal{D}}_{t}^{k}:=\sigma\left(\mathbf{1}_{\left\{\tau_{k} \leq s\right\}}, s \in[0, t]\right)$. Note that under the assumption of the independence of the vector $\boldsymbol{\tau}$ and the financial market, the process $W$ remains a $\mathbb{G}$-Brownian motion, see El Karoui et al. (2013) for more details.

Let $\mathbb{P}\left(\tau_{i}<.\right)$ be the marginal distribution of the r.v. $\tau_{i}$. We assume that the death times are dependent and propose to link the marginal distributions to their joint distribution using 
a Farlie-Gumbel-Morgenstern (FGM) copula family defined as:

$$
C_{\boldsymbol{\theta}}\left(u_{1}, \ldots, u_{n}\right)=\prod_{i=1}^{n} u_{i}\left[1+\sum_{k=2}^{n} \sum_{1 \leq j_{1} \leq \ldots j_{k} \leq n} \theta_{j_{1} \ldots j_{k}}\left(1-u_{j_{1}}\right) \ldots\left(1-u_{j_{k}}\right)\right] \text {, }
$$

with $\left(u_{1}, \ldots, u_{n}\right) \in[0,1]^{n}$ and $-1 \leq \theta_{j_{1} \ldots j_{k}} \leq 1$ for all $j_{1}, \ldots, j_{k}$, see Jaworski et al. (2010, page 19). In other words, we have

$$
\mathbb{P}\left(\tau_{1}<u_{1}, \ldots, \tau_{n}<u_{n}\right)=C_{\boldsymbol{\theta}}\left(\mathbb{P}\left(\tau_{1}<u_{1}\right), \ldots, \mathbb{P}\left(\tau_{n}<u_{n}\right)\right), \quad\left(u_{1}, \ldots, u_{n}\right) \in \mathbb{R}^{n},
$$

For the sake of applicability, we let each random variable $\tau_{i}$ have an exponential marginal distribution with parameters $\lambda_{i}$. The following result gives a closed form formula for the joint density $\gamma$ of $\left(\tau_{1}, \ldots, \tau_{n}\right)$.

Lemma 1. Let $\left(u_{1}, \ldots, u_{n}\right) \in \mathbb{R}_{+}^{n}$. The joint density is given by

$$
\frac{1}{S} \gamma\left(u_{1}, \ldots, u_{n}\right)=\sum_{k=1}^{n} \sum_{1 \leq j_{1} \leq \cdots \leq j_{k} \leq n}(-1)^{n} d_{j_{1} \ldots j_{k}} e^{-\lambda_{j_{1}} u_{j_{1}}-\cdots-\lambda_{j_{k}} u_{j_{k}}}+d,
$$

where $S=(-1)^{n} \prod_{i=1, \ldots, n} \lambda_{i} e^{-\lambda_{i} u_{i}}$ and

$$
\begin{aligned}
d_{j_{1}} & =2\left[(-1)^{n-1} \sum_{i_{1} \neq j_{1}} \theta_{j_{1} i_{1}}+(-1)^{n-2} \sum_{i_{1}, i_{2} \neq j_{1}} \theta_{j_{1} i_{1} i_{2}} \cdots+\sum_{i_{1} \ldots i_{n-2} \neq j_{1}} \theta_{j_{1} i_{1} \ldots i_{n-2}}-\theta_{1 \ldots n}\right], \\
d_{j_{1} j_{2}} & =4\left[(-1)^{n} \theta_{j_{1} j_{2}} \cdots-\sum_{i_{1} \ldots i_{n-3} \neq j_{1}} \theta_{j_{1} j_{2} i_{1} \ldots i_{n-3}}+\theta_{1 \ldots n}\right], \\
d_{j_{1} j_{2} j_{3}} & =8\left[(-1)^{n} \theta_{j_{1} j_{2} j_{3}} \cdots+\sum_{i_{1} \ldots i_{n-3} \neq j_{1}} \theta_{j_{1} j_{2} j_{3} i_{1} \ldots i_{n-4}}-\theta_{1 \ldots n}\right], \\
\vdots & \\
d_{j_{1} \ldots j_{n}} & =(-2)^{n} \theta_{1 \ldots n}, \quad \sum_{1 \leq i_{1} \cdots \leq i_{n-2} \leq n} \theta_{i_{1} \ldots i_{n-2}}-\sum_{1 \leq i_{1} \ldots \leq i_{n-1} \leq n} \theta_{i_{1} \ldots i_{n-1}}+\theta_{1 \ldots n .} .
\end{aligned}
$$

The conditional density of death time $\tau_{1}$ conditional on $\tau_{2}, \ldots, \tau_{n}$ defined as

$$
\gamma\left(u_{1} \mid u_{2}, \ldots, u_{n}\right)=\frac{\gamma\left(u_{1}, \ldots, u_{n}\right)}{\int_{0}^{\infty} \gamma\left(u_{1}, \ldots, u_{n}\right) d u_{1}}
$$

with the denominator given by the explicit formula

$$
\begin{aligned}
\int_{0}^{\infty} \gamma\left(u_{1}, \ldots, u_{n}\right) d u_{1} & =e^{\lambda_{1} u_{1}}\left(\sum_{k=1}^{n} \sum_{2 \leq j_{1} \leq \ldots j_{k} \leq n} d_{j_{1} \ldots j_{k}} e^{-\lambda_{j_{1}} u_{j_{1}}-\cdots-\lambda_{j_{k}} u_{j_{k}}}+d+\frac{d_{1}}{2}\right. \\
& \left.+\frac{1}{2} \sum_{k=1}^{n-1} \sum_{2 \leq j_{1} \leq \ldots j_{k} \leq n} d_{1 j_{1} \ldots j_{k}} e^{-\lambda_{j_{1}} u_{j_{1}}-\cdots-\lambda_{j_{k}} u_{j_{k}}}\right) .
\end{aligned}
$$


Proof. Following standard calculations, we easily find the results.

For each $i \in\{1, \cdots, n\}$, we introduce the death indicator process $H^{i}=\left(H_{t}^{i}\right)_{t \geq 0}$ defined as $H_{t}^{i}:=\mathbf{1}_{\left\{\tau_{i} \leq t\right\}}$ for all $t \geq 0$. The following result ensures the existence of the $\mathbb{G}$-intensities (see Kharroubi and Lim (2014)).

Proposition 1. For all $i=1, \cdots, n, H_{t}^{i}$ admits a $\mathbb{G}$-intensity $\lambda_{t}^{i}$ such that $H_{t}^{i}-\int_{0}^{t \wedge \tau_{i}} \lambda_{s}^{i} d s$ is $a \mathbb{G}$-martingale.

In our context the intensity $\lambda^{i}$ is known as mortality intensity and is bounded. The dynamics of the intensities $\lambda_{t}^{i}$, for $i=1, \cdots, n$, can be completely deduced from the joint density, see El Karoui et al. (2013). In the particular case of two individuals, we can easily write

$$
\begin{aligned}
& \lambda_{t}^{1}=\mathbf{1}_{\left\{\tau_{2}>t\right\}} \frac{\int_{t}^{\infty} \gamma\left(t, \theta_{2}\right) d \theta_{2}}{\int_{t}^{\infty} \int_{\theta_{1}}^{\infty} \gamma\left(\theta_{1}, \theta_{2}\right) d \theta_{1} d \theta_{2}}+\mathbf{1}_{\left\{\tau_{2} \leq t\right\}} \frac{\gamma\left(t, \tau_{2}\right)}{\int_{t}^{\infty} \gamma\left(\theta_{1}, \tau_{2}\right) d \theta_{1}}, \\
& \lambda_{t}^{2}=\mathbf{1}_{\left\{\tau_{1}>t\right\}} \frac{\int_{t}^{\infty} \gamma\left(\theta_{1}, t\right) d \theta_{1}}{\int_{t}^{\infty} \int_{\theta_{1}}^{\infty} \gamma\left(\theta_{1}, \theta_{2}\right) d \theta_{1} d \theta_{2}}+\mathbf{1}_{\left\{\tau_{1} \leq t\right\}} \frac{\gamma\left(\tau_{1}, t\right)}{\int_{t}^{\infty} \gamma\left(\tau_{1}, \theta_{2}\right) d \theta_{2}}
\end{aligned}
$$

The above framework will serve to characterize the dependence structure between individuals' lifetimes, which are due, for example, to medical breakthroughs or environmental factors that affect a whole population. These play a crucial role in the risk management for life insurance risks. Moreover, the density approach provides a new vision for modeling the dependency of deaths among a population not only from a theoretical point of view but also for a practical use. Remark that (1.3) amount to saying that one individual's intensity will have a jump when the other individual is deceased. More precisely, the dependence effect arises due to the fact that the arrival of first death helps localize the other death occurrence in the support of its conditional law. Similar interpretation can also be drawn when it comes to the characterization of a portfolio of $n$ individuals. In this case, we can also derive a similar closed form expression of the intensities as in (1.3). On the other hand, the density framework encompasses the traditional case of (conditionally) independent lives, see El Karoui et al. (2013). The conditional independence can arise, for example, when death occurrences share some common random factors. This is the case, for example, of the celebrated Carter and Lee (1992) model which introduce a dependence on the mortality intensities for a given population through a shared Gaussian factor.

1.3 Strategies and utility function. The insurer can trade on the above assets and her trading strategy is defined as a $\mathbb{G}^{n}$-predictable process $\pi=\left(\pi_{t}\right)_{0 \leq t \leq T}$ when $\int \pi \frac{d S}{S}$ is welldefined where $S=\left(S_{t}\right)_{t \in[0, T]}$ the discounted value of $\hat{S}$ is defined as $S_{t}=e^{-r t} \hat{S}_{t}$. The process $\pi$ describes the discounted amount of money invested in the portfolio of risky assets. Assuming that the investment strategy of the insurer is self-financed and denoted $X^{\pi}=\left(X_{t}^{\pi}\right)_{t \in[0, T]}$, the discounted value of the insurer portfolio with initial capital 0 and following the strategy $\pi$, can be described as:

$$
X_{t}^{\pi}=\int_{0}^{t} \pi_{s}\left(\mu_{s}-r\right) d s+\int_{0}^{t} \pi_{s} \sigma_{s} d W_{s}
$$


We denote $X_{t}^{x, \pi}$ as the discounted wealth at time $t$, when the initial capital $x \geq 0$ and the investment strategy is $\pi$. We will assume that the insurance company aims at maximizing the expected value of the utility of her terminal wealth at time $T$. Thus, let $U$ be the exponential utility function with risk aversion coefficient $\alpha>0$, that is:

$$
\forall y \in \mathbb{R}, \quad U(y)=-e^{-\alpha y} .
$$

In the following definition, we introduce the set of admissible strategies for the insurer, making usual restrictions that ensure the existence and some integrability properties for the processes involved.

Definition 1. For any $\mathbb{G}^{n}$-stopping times $w$ and $v$ satisfying $0 \leq w \leq v \leq T$, the set of admissible trading strategies $\mathcal{A}_{\mathbb{G}^{n}}[w, v]$ consists of all $\mathbb{G}^{n}$-predictable processes $\pi=\left(\pi_{t}\right)_{w \leq t \leq v}$ which satisfy

$$
\mathbb{E}\left[\int_{w}^{v}\left|\pi_{t}\right|^{2} d t\right]<\infty
$$

and $\left\{\exp \left(-\alpha X_{\vartheta}^{\pi}\right), \vartheta\right.$ is a $\mathbb{G}^{n}$-stopping time with values in $\left.[w, v]\right\}$ is an uniformly integrable family.

\section{Utility maximization and indifference pricing}

The main objective of this section is to characterize the optimal price $p^{*}$, if it exists, which makes the insurer indifferent between not selling a new portfolio with $m$ contracts, as described above, and selling it for the price $p^{*}$. For this purpose, let $\tau_{n+i}$, for $i=1, \ldots, m$, be the death time of an individual in the new portfolio (which will be sold). Then, let $V(x)$ and $\tilde{V}(x)$ be the value functions associated with the maximum expected utility problem and defined for $x \geq 0$ as

$$
V(x):=\sup _{\pi \in \mathcal{A}_{\mathbb{G}} n\left[0, T \wedge\left(\tau_{1} \vee \cdots \vee \tau_{n}\right)\right]} \mathbb{E}\left[U\left(x+X_{T \wedge\left(\tau_{1} \vee \cdots \vee \tau_{n}\right)}^{\pi}-\sum_{j=1}^{n} F_{T \wedge \tau_{j}}^{j}\right)\right],
$$

and

$$
\tilde{V}(x):=\sup _{\pi \in \mathcal{A}_{\mathbb{G} n+m}\left[0, T \wedge\left(\tau_{1} \vee \cdots \vee \tau_{n+m}\right)\right]} \mathbb{E}\left[U\left(x+X_{T \wedge\left(\tau_{1} \vee \cdots \vee \tau_{n+m}\right)}^{\pi}-\sum_{j=1}^{n+m} F_{T \wedge \tau_{j}}^{j}\right)\right] .
$$

Then, the optimal price $p^{*}$ that makes the insurer indifferent towards such an additional exposure is given as:

$$
p^{*}=\inf \{p>0, V(x) \leq \tilde{V}(x+p)\} .
$$

Whenever a solution of the above problem exists, it will be called an indifference price. Notice that the latter will not depend on the initial wealth $x$, invested by the insurer, since the utility function is the exponential function. This can be easily seen from the special multiplicative factoring of $x$ in the expressions of $V(x)$ and $\tilde{V}(x)$ above. Therefore, in what 
follows we consider the case $x=0$ and for simplicity of notation, we write $V$ (resp. $\tilde{V}$ ), instead of $V(0)$ (resp. $\tilde{V}(0))$.

The case of selling a new portfolio can be thought as if the insurer already holds a portfolio of termed insurance contracts seeks, for example, to buy an entire second portfolio with the same termination date $T$. An appealing example, may be borrowed from reinsurance industry. Generally, reinsurers are massively exposed to mortality risk. Inherent in some pure endowment or death benefit contracts, they reinsure and seek to purchase longevity-sensitive contracts, for e.g. temporary life annuities, in order to hedge out the initial exposure to mortality. Moreover, mixing different pure life insurance may also motivate such an acquisition, see e.g. Marceau and Gaillardetz (1999), Milevsky and Promislow (2001) and Cairns et al. (2004) which investigate the issue of natural hedging considered also in Cox and Lin (2007). The former explores the impact of mortality changes on life insurance and annuities separately, or investigates a simple combination of life and pure endowment life contracts.

Remark 1. If we consider $n$ contracts in the first portfolio held by the reinsurer and $m$ in the second one, the indifference price could be determined using the backward stochastic differential equations (BSDE) tools. This is based on generalization of the result of Kharroubi and Lim (2011, 2012) when $m=1$ and $n=0$. However, as we may see later, it is difficult to use these results in numerical analyzes (too long to compute). Since the death times are not ordered, to compute $\tilde{V}$, we need to solve $(n-1) n$ ! BSDEs (for more details see Appendix A.1). Therefore, for the sake of applicability, we propose to reduce the dimensionality of our problem by replacing each portfolio by a representative contract.

2.1 Representative contract construction. Here, we propose to replace each portfolio by a representative contract. Such an idea is widely used in practical applications. Indeed, it is common to consider grouping policies into model cells and replace all policies in each group with a representative policy, see Goffard and Guerrault (2015). Recall that each portfolio is composed of contracts not only of different guarantees, but also of individuals of ages and hence different mortality profiles. Therefore, the representative contracts are characterized by a death time and a guaranteed amount. To find the representative contracts for the two portfolios, we consider a virtual world on which we define two random times $\tau$ and $\tilde{\tau}$ (the representative agents' death times ) satisfying the following conditions.

\section{Assumption 2.}

(i) $\tau$ and $\tilde{\tau}$ are exponentially-distributed r.v.'s with intensity $\lambda$ and $\tilde{\lambda}$ respectively;

(ii) $\tau$ is independent of $\left(\tau_{i}, i=1 \ldots, n\right)$;

(iii) $\tilde{\tau}$ is independent of $\left(\tau_{i}, i=n+1, \ldots, n+m\right)$;

(iv) Let c (resp. $\tilde{c})$ be the representative endowment for the first (resp. second) portfolio, defined as follows:

$$
c=c_{1} g\left(\lambda_{1}\right)+\cdots+c_{n} g\left(\lambda_{n}\right),
$$

with $g(x)=\frac{x}{x+r}\left(1-e^{-(x+r) T}\right)$. Note that $\tilde{c}$ is given by a similar formula; 
(v) $\tau$ and $\tilde{\tau}$ are correlated by a FGM copula.

These assumptions can be read as follows. First, (ii) and (iii) amount to seeing that the representative agent for each portfolio evolves in a virtual world. That is, the representative contract is completely independent of the initial portfolio and it is only linked to the latter through its specific characteristic. In assumption (iv) we require that the representative agent reproduces the guaranteed amount of the considered portfolio. This aggregated amount is weighted by $g\left(\lambda_{i}\right)=\mathbb{E}\left[e^{-r \tau_{i}} \mathbf{1}_{\left\{\tau_{i}<T\right\}}\right]$ in order to reproduce the expected discounted exposure of the insurer. Since we consider a virtual world, we have the independence between the representative agents' death times $(\tau$ and $\tilde{\tau})$ and the death time $\tau_{i}$ of each agent of the initial group, with $i=1, \ldots, n$ for the first group and $i=n+1, \ldots, n+m$ for the second one. However, in order to maintain the same correlation between the initial portfolios, the two random times $\tau$ and $\tilde{\tau}$ will be correlated. The correlation translates the initial portfolios correlation and it is defined by an FGM copula (assumption (v)).

To fully characterize the representative agents, we should determine their mortality profiles. Thus, we are looking for the intensities which lead to having the same risk (of the representative contract and the portfolio) and an expected cash-flows as close as possible to the initial portfolio. In the following, we detail the computation of $\lambda$ (the same reasoning applies to find $\tilde{\lambda}$ ). Since the risk of the initial portfolio and the required contract are the same, then $\lambda$ is solution of

$$
f(\lambda)=\frac{1}{c^{2}} \operatorname{Var}\left[\sum_{i=1}^{n} c_{i} e^{-r \tau_{i}} \mathbf{1}_{\left\{\tau_{i}<T\right\}}\right]
$$

where

$$
f(x)=\operatorname{Var}\left[e^{-r \tau} \mathbf{1}_{\{\tau<T\}}\right]=\frac{x}{x+2 r}\left(1-e^{-(x+2 r) T}\right)-\left(\frac{x}{x+r}\right)^{2}\left(1-e^{-(x+r) T}\right)^{2},
$$

and $c$ defined as in Assumption 2.

Equation (2.2) has two solutions $l_{1}$ and $l_{2}$ such that $l_{1} \leq \lambda^{*} \leq l_{2}$, where the function $f$ reaches its maximum at $\lambda^{*}$. Using the independence hypothesis between $\tau$ and $\left(\tau_{i}, i=1, \ldots, n\right)$, we choose $l_{i}, i=1$ or 2 , which minimizes the expected present value of the insurer portfolio's liability, i.e. $\left|\mathbb{E}\left[\sum_{i=1}^{n} c_{i} e^{-r \tau_{i}} \mathbf{1}_{\left\{\tau_{i}<T\right\}}\right]-\mathbb{E}\left[c e^{-r \tau} \mathbf{1}_{\{\tau<T\}}\right]\right|$. It is easy to verify that our problem has an unique solution since $\lambda \rightarrow \mathbb{E}\left[e^{-r \tau} \mathbf{1}_{\{\tau<T\}}\right]=g(\lambda)$ is an increasing function. Consequently, given the intensity $\lambda$, we define $\tau$ as

$$
\tau=\inf \{t>0: \lambda t \geq X\}
$$

where $X$ is an exponential random variable with parameter equal to 1 , i.e. $\mathbb{P}(X \geq t)=e^{-t}$.

Next, we characterize the correlation between $\tau$ and $\tilde{\tau}$. The two random times are correlated by the FGM copula given by

$$
c(u, v)=u v[1+\theta(1-u)(1-v)], \quad \theta \in[-11], \quad(u, v) \in[0,1]^{2} .
$$

Therefore, we choose $\theta$ so that the variance of the aggregate portfolio, with the additional $m$ policyholders, is best replicated by the addition of the representative agents. Formally, the 
coefficient $\theta$ is set as the solution of the minimization problem:

$$
\min _{\theta \in[-11]}\left|\operatorname{Var}\left(\sum_{i=1}^{n+m} c_{i} e^{-r \tau_{i}} \mathbf{1}_{\left\{\tau_{i}<T\right\}}\right)-\operatorname{Var}\left(c e^{-r \tau} \mathbf{1}_{\{\tau<T\}}+\tilde{c} e^{-r \tilde{\tau}} \mathbf{1}_{\{\tilde{\tau}<T\}}\right)\right|,
$$

where $\tilde{c}=\sum_{i=1}^{m} c_{n+i} g\left(\lambda_{n+i}\right)$, see Assumption 2. The latter is equivalent to

$$
\min _{\theta \in[-11]}\left|\operatorname{Cov}\left(\sum_{i=1}^{n} c_{i} e^{-r \tau_{i}} \mathbf{1}_{\left\{\tau_{i}<T\right\}}, \sum_{i=n+1}^{n+m} c_{i} e^{-r \tau_{i}} \mathbf{1}_{\left\{\tau_{i}<T\right\}}\right)-\operatorname{Cov}\left(c e^{-r \tau} \mathbf{1}_{\{\tau<T\}}, \tilde{c} e^{-r \tilde{\tau}} \mathbf{1}_{\{\tilde{\tau}<T\}}\right)\right| .
$$

In other words, the optimal correlation coefficient $\theta$ is that which allows for best replication of the initial correlation between the two portfolios. It is worth mentioning that by doing so we also ensure that the optimal parameter $\theta$ will capture the sign of the dependency between the two portfolios, especially negative correlations. This latter property is of paramount importance in view of the initial problem of natural hedging between biometric risks.

Henceforth, remarking that $\mathbb{E}\left(e^{-r \tau} \mathbf{1}_{\{\tau<T\}} e^{-r \tilde{\tau}} \mathbf{1}_{\{\tilde{\tau}<T\}}\right)$ is a linear function of $\theta$, we find that $\operatorname{Cov}\left(c e^{-r \tau} \mathbf{1}_{\{\tau<T\}}, \tilde{c} e^{-r \tilde{\tau}} \mathbf{1}_{\{\tilde{\tau}<T\}}\right)=c \tilde{c} A_{1} \theta$ where

$$
\begin{aligned}
A_{1}= & \lambda \tilde{\lambda}\left[\frac{\left(1-e^{-(\lambda+r) T}\right)\left(1-e^{-(\tilde{\lambda}+r) T}\right)}{(r+\lambda)(\tilde{\lambda}+r)}+4 \frac{\left(1-e^{-(2 \lambda+r) T}\right)\left(1-e^{-(2 \tilde{\lambda}+r) T}\right)}{(r+2 \lambda)(2 \tilde{\lambda}+r)}\right. \\
& \left.-2 \frac{\left(1-e^{-(2 \lambda+r) T}\right)\left(1-e^{-(\tilde{\lambda}+r) T}\right)}{(r+2 \lambda)(\tilde{\lambda}+r)}-2 \frac{\left(1-e^{-(\lambda+r) T}\right)\left(1-e^{-(2 \tilde{\lambda}+r) T}\right)}{(r+\lambda)(2 \tilde{\lambda}+r)}\right] .
\end{aligned}
$$

Finally, our problem $\left.\min _{\theta \in[-1} 1\right]\left|\operatorname{Cov}\left(\sum_{i=1}^{n} c_{i} e^{-r \tau_{i}} \mathbf{1}_{\left\{\tau_{i}<T\right\}}, \sum_{i=n+1}^{n+m} c_{i} e^{-r \tau_{i}} \mathbf{1}_{\left\{\tau_{i}<T\right\}}\right)-c \tilde{c} A_{1} \theta\right|$ has an unique solution.

Remark 2. If the death times $\tau_{i}, i=1, \ldots, n+m$ are correlated by the FGM copula given by Equation (1.1), i.e.

$$
C\left(u_{1}, \ldots, u_{n+m}\right)=\prod_{i=1}^{n+m} u_{i}\left[1+\sum_{k=2}^{n+m} \sum_{1 \leq j_{1} \leq \ldots j_{k} \leq d} \theta_{j_{1} \ldots j_{k}}\left(1-u_{j_{1}}\right) \ldots\left(1-u_{j_{k}}\right)\right],
$$

then the covariance between the initial portfolios is

$$
\begin{aligned}
& \operatorname{Cov}\left(\sum_{i=1}^{n} c_{i} e^{-r \tau_{i}} \mathbf{1}_{\left\{\tau_{i}<T\right\}}, \sum_{i=n+1}^{n+m} c_{i} e^{-r \tau_{i}} \mathbf{1}_{\left\{\tau_{i}<T\right\}}\right) \\
& =\sum_{i=1}^{n} \sum_{j=n+1}^{n+m} \theta_{i j} c_{i} c_{j}\left(\frac{\lambda_{i}\left(1-e^{-\left(r+\lambda_{i}\right) T}\right)}{\lambda_{i}+r}-2 \frac{\lambda_{i}\left(1-e^{-\left(r+2 \lambda_{i}\right) T}\right)}{2 \lambda_{i}+r}\right) \\
& \times\left(\frac{\lambda_{j}\left(1-e^{-\left(r+\lambda_{j}\right) T}\right)}{\lambda_{j}+r}-2 \frac{\lambda_{j}\left(1-e^{-\left(r+2 \lambda_{j}\right) T}\right)}{2 \lambda_{j}+r}\right)
\end{aligned}
$$

Remark 3. So far, the model points construction considered is applied to portfolios of death benefit policies. Note that the same procedure can also be used to represent portfolios with heterogeneous life insurance contracts. An appealing example is the pure endowment contracts 
that pays out a lump sum at time $T$ if the individual is alive at this time.

2.2 Optimization problem with two policies. Since each portfolio may be viewed as a contract, we present here the indifference pricing of two contracts. The general case is detailed in Appendix A.1. Recall that in order to find the indifference price, we shall compute the following quantities

$$
V:=\sup _{\pi \in \mathcal{A}_{\mathbb{G}^{1}}[0, T]} \mathbb{E}\left[U\left(X_{T \wedge \tau_{1}}^{\pi}-F_{T \wedge \tau_{1}}\right)\right]
$$

and

$$
\tilde{V}:=\sup _{\pi \in \mathcal{A}_{\mathbb{G}^{2}}[0, T]} \mathbb{E}\left[U\left(X_{T \wedge\left(\tau_{1} \vee \tau_{2}\right)}^{\pi}-F_{T \wedge \tau_{1}}^{1}-F_{T \wedge \tau_{2}}^{2}\right)\right]
$$

The Equation (2.3) corresponds to the value of the maximum expected utility of the wealth at time $T$ when the insurance company has sold one policy. In the absence of a second contract, the $\tau_{1}$-intensity is given by $\frac{\gamma(t)}{\int_{t}^{\infty} \gamma(s) d s}$ where $\gamma$ is the density of $\tau_{1}$. The following propositions give the $V$ and $\tilde{V}$ solutions of the optimization problem in terms of the initial value of a BSDE.

Proposition 2. The value function $V:=\sup _{\pi \in \mathcal{A}_{\mathbb{G}^{1}}[0, T]} \mathbb{E}\left[U\left(X_{T}^{\pi}\right)\right]$ is given by

$$
V=-\exp \left(\alpha y_{0}\right)
$$

where $(y, z, u)$ is the solution of the BSDE

$$
y_{t \wedge \tau_{1}}=F_{t \wedge \tau_{1}}^{1}+\int_{t \wedge \tau_{1}}^{T \wedge \tau_{1}} h\left(s, z_{s}, u_{s}\right) d s-\int_{t \wedge \tau_{1}}^{T \wedge \tau_{1}} z_{s} d W_{s}-\int_{t \wedge \tau_{1}}^{T \wedge \tau_{1}} u_{s} d H_{s}^{1}, \quad 0 \leq t \leq T,
$$

where $h(s, z, u)=\frac{e^{\alpha u}-1}{\alpha} \frac{\gamma(t)}{\int_{t}^{\infty} \gamma(s) d s}-z \frac{\left(\mu_{t}-r\right)}{\sigma t}-\frac{1}{2 \alpha} \frac{\left(\mu_{t}-r\right)^{2}}{\sigma_{t}^{2}}$.

Moreover, the optimal strategy $\pi_{t}^{*}$ associated to this problem is defined for each $t \in[0, T]$ by

$$
\pi_{t}^{*}=\frac{1}{\sigma_{t}}\left(z_{t}+\frac{\mu_{t}-r}{\alpha \sigma_{t}}\right)
$$

and $y_{t}=y_{t}^{0} \mathbf{1}_{\left\{t<\tau_{1}\right\}}+F_{\tau_{1}}^{1} \mathbf{1}_{\left\{\tau_{1} \leq t\right\}}$ with $y_{t}^{0}=F_{T}^{1}+\int_{t}^{T} h\left(s, z_{s}^{0}, F_{s}^{1}-y_{s}^{0}\right) d s-\int_{t}^{T} z_{s}^{0} d W_{s}$.

Proof. This follows directly from $\mathrm{Hu}$ et al. (2005).

We will now turn to the case where the insurance company sells a second contract. We recall that the corresponding value function associated to the maximum expected utility is given by (2.4).

Proposition 3. Under the assumptions of Section 1, the function $\tilde{V}$ is given by $\tilde{V}(x)=$ $-\exp \left(\alpha Y_{0}\right)$ where $Y_{0}$ is the initial value of the unique solution $(Y, Z, U)$ of the BSDE

$$
Y_{t}=B+\int_{t \wedge\left(\tau_{1} \vee \tau_{2}\right)}^{T \wedge\left(\tau_{1} \vee \tau_{2}\right)} f\left(s, Z_{s}, U_{s}^{1}, U_{s}^{2}\right) d s-\int_{t \wedge\left(\tau_{1} \vee \tau_{2}\right)}^{T \wedge\left(\tau_{1} \vee \tau_{2}\right)} Z_{s} d W_{s}-\int_{t \wedge\left(\tau_{1} \vee \tau_{2}\right)}^{T \wedge\left(\tau_{1} \vee \tau_{2}\right)}\left(U_{s}^{1} d H_{s}^{1}+U_{s}^{2} d H_{s}^{2}\right)
$$


for $t \in[0, t]$, with $f\left(t, z, u_{1}, u_{2}\right)=\sum_{i=1,2}\left(1-H_{t}^{i}\right) \frac{e^{\alpha u^{i}}-1}{\alpha} \lambda_{t}^{i}-z \frac{\left(\mu_{t}-r\right)}{\sigma_{t}}-\frac{1}{2 \alpha} \frac{\left(\mu_{t}-r\right)^{2}}{\sigma_{t}^{2}}$ and $\lambda^{1}, \lambda^{2}$ given by $(1.3)$.

Moreover, there exists an optimal strategy $\hat{\pi}^{*}$ given by

$$
\hat{\pi}_{t}^{*}=\frac{1}{\sigma_{t}}\left(Z_{t}+\frac{\mu_{t}-r}{\alpha \sigma_{t}}\right) .
$$

Proof. See Appendix A.2.

Subsequently, the optimal price solution of the Equation (2.1), making the insurer indifferent to sell or not the additional contract, is given in terms of the solutions of the two BSDEs in the above propositions.

Corollary 1. The indifference price is

$$
p^{*}=\frac{1}{\alpha} \ln \left(\frac{\tilde{V}}{V}\right)=y_{0}-Y_{0} .
$$

To numerically determine $p^{*}$, we should simulate the BSDEs in Proposition 2 and Proposition 3. The former is straightforward and can be simulated using classical schemes. However, the second one related to $(Y, Z, U)$ needs some further arrangement as it is a BSDE with random jumps. Therefore, let us give more details for the BSDE in Proposition 3. For $t \in[0, T]$, we consider the following BSDE :

$$
Y_{t}=B+\int_{t \wedge\left(\tau_{1} \vee \tau_{2}\right)}^{T \wedge\left(\tau_{1} \vee \tau_{2}\right)} f\left(s, Z_{s}, U_{s}^{1}, U_{s}^{2}\right) d s-\int_{t \wedge\left(\tau_{1} \vee \tau_{2}\right)}^{T \wedge\left(\tau_{1} \vee \tau_{2}\right)} Z_{s} d W_{s}-\int_{t \wedge\left(\tau_{1} \vee \tau_{2}\right)}^{T \wedge\left(\tau_{1} \vee \tau_{2}\right)}\left(U_{s}^{1} d H_{s}^{1}+U_{s}^{2} d H_{s}^{2}\right) .
$$

Considering the order of the deaths $\tau_{1}$ and $\tau_{2}$ and decomposing the related death indicators $H_{t}^{1}$ and $H_{t}^{2}$, we notice that the generator $f$ of the above BSDE can be written as follows:

$$
\begin{aligned}
f\left(t, z, u_{1}, u_{2}\right)=\mathbf{1}_{\left\{t<\tau_{1} \wedge \tau_{2}\right\}}\left[\sum_{i=1,2} \frac{e^{\alpha u_{i}}-1}{\alpha} \lambda_{t}^{i}-z \frac{\left(\mu_{t}-r\right)}{\sigma_{t}}-\frac{1}{2 \alpha} \frac{\left(\mu_{t}-r\right)^{2}}{\sigma_{t}^{2}}\right] \\
+\mathbf{1}_{\left\{\tau_{1} \leq t<\tau_{2}\right\}}\left[\frac{e^{\alpha u_{2}}-1}{\alpha} \lambda_{t}^{2}-z \frac{\left(\mu_{t}-r\right)}{\sigma_{t}}-\frac{1}{2 \alpha} \frac{\left(\mu_{t}-r\right)^{2}}{\sigma_{t}^{2}}\right] \\
+\mathbf{1}_{\left\{\tau_{2} \leq t<\tau_{1}\right\}}\left[\frac{e^{\alpha u_{1}}-1}{\alpha} \lambda_{t}^{1}-z \frac{\left(\mu_{t}-r\right)}{\sigma_{t}}-\frac{1}{2 \alpha} \frac{\left(\mu_{t}-r\right)^{2}}{\sigma_{t}^{2}}\right] \\
+\mathbf{1}_{\left\{\tau_{2} \vee \tau_{1} \leq t\right\}}\left[-z \frac{\left(\mu_{t}-r\right)}{\sigma_{t}}-\frac{1}{2 \alpha} \frac{\left(\mu_{t}-r\right)^{2}}{\sigma_{t}^{2}}\right] .
\end{aligned}
$$

The terminal value $B$ of $Y$ should be decomposed similarly depending on the ranking of the death times.

\section{Assumption 3.}

1. The terminal value of the $B S D E$ in Proposition $3 B \in \mathcal{G}_{\mathcal{T}}$, can be written as follows $B=B^{0} \mathbf{1}_{\left\{T<\tau_{1} \wedge \tau_{2}\right\}}+B^{1}\left(\tau_{1}\right) \mathbf{1}_{\left\{\tau_{1} \leq T<\tau_{2}\right\}}+B^{2}\left(\tau_{2}\right) \mathbf{1}_{\left\{\tau_{2} \leq T<\tau_{1}\right\}}+B^{3}\left(\tau_{1}, \tau_{2}\right) \mathbf{1}_{\left\{\tau_{1} \vee \tau_{2} \leq T\right\}}$, with $B^{0}=F_{T}^{1}+F_{T}^{2}, B^{1}\left(\tau_{1}\right)=F_{\tau_{1}}^{1}+F_{T}^{2}, B^{2}\left(\tau_{2}\right)=F_{T}^{1}+F_{\tau 2}^{2}$ and $B^{3}\left(\tau_{1}, \tau_{2}\right)=F_{\tau_{1}}^{1}+F_{\tau_{2}}^{2}$. 
2. Given the decomposition in Equation (2.5), the generator $f$ can be written as

$$
\begin{array}{r}
f\left(t, y, z, u_{1}, u_{2}\right) \mathbf{1}_{\left\{t \leq \tau_{1} \vee \tau_{2}\right\}}=f^{0}\left(t, y, z, u_{1}, u_{2}\right) \mathbf{1}_{\left\{t<\tau_{1} \wedge \tau_{2}\right\}}+f^{1}\left(t, y, z, u_{1}, u_{2}, \tau_{1}\right) \mathbf{1}_{\left\{\tau_{1} \leq t<\tau_{2}\right\}} \\
+f^{2}\left(t, y, z, u_{1}, u_{2}, \tau_{2}\right) \mathbf{1}_{\left\{\tau_{2} \leq t<\tau_{1}\right\}}+f^{3}\left(t, y, z, u_{1}, u_{2}, \tau_{1}, \tau_{2}\right) \mathbf{1}_{\left\{\tau_{1} \vee \tau_{2} \leq t\right\}} .
\end{array}
$$

3. We assume that the following BSDEs admit a solution

$$
\begin{gathered}
\left\{\begin{aligned}
Y_{t}^{1}\left(\theta_{1}\right)= & B^{1}\left(\theta_{1}\right)+\int_{t}^{T} f^{1}\left(s, Y_{s}^{1}\left(\theta_{1}\right), Z_{s}^{1}\left(\theta_{1}\right), 0, B^{3}\left(\theta_{1}, s\right)-Y_{s}^{1}\left(\theta_{1}\right), \theta_{1}\right) d s \\
& \quad-\int_{t}^{T} Z_{s}^{1}\left(\theta_{1}\right) d W_{s}, \\
Y_{t}^{0,1}= & B^{0}+\int_{t}^{T} f^{0}\left(s, Y_{s}^{0,1}, Z_{s}^{0,1}, Y_{s}^{1}(s)-Y_{s}^{0,1}, 0\right) d s-\int_{t}^{T} Z_{s}^{0,1} d W_{s},
\end{aligned}\right. \\
\left\{\begin{aligned}
Y_{t}^{2}\left(\theta_{2}\right)= & B^{2}\left(\theta_{2}\right)+\int_{t}^{T} f^{2}\left(s, Y_{s}^{2}\left(\theta_{2}\right), Z_{s}^{2}\left(\theta_{2}\right), B^{3}\left(s, \theta_{2}\right)-Y_{s}^{2}\left(\theta_{2}\right), 0, \theta_{2}\right) d s \\
& \quad-\int_{t}^{T} Z_{s}^{2}\left(\theta_{2}\right) d W_{s}, \\
Y_{t}^{0,2}= & B^{0}+\int_{t}^{T} f^{0}\left(s, Y_{s}^{0,2}, Z_{s}^{0,2}, 0, Y_{s}^{2}(s)-Y_{s}^{0,2}\right) d s-\int_{t}^{T} Z_{s}^{0,2} d W_{s} .
\end{aligned}\right.
\end{gathered}
$$

Under the above assumption, the solution of the BSDE in Proposition 3 with two random jumps $\tau_{1}$ and $\tau_{2}$ can be decomposed into two auxiliary Brownian BSDEs depending on the order these jumps as follows:

$$
\left\{\begin{aligned}
Y_{t}= & \mathbf{1}_{\left\{\tau_{2}<\tau_{1}\right\}}\left(Y_{t}^{0,2} \mathbf{1}_{\left\{t<\tau_{2}\right\}}+Y_{t}^{2}\left(\tau_{2}\right) \mathbf{1}_{\left\{\tau_{2} \leq t<\tau_{1}\right\}}+B^{3}\left(\tau_{1}, \tau_{2}\right) \mathbf{1}_{\left\{\tau_{2}<\tau_{1} \leq t\right\}}\right) \\
& \quad+\mathbf{1}_{\left\{\tau_{1}<\tau_{2}\right\}}\left(Y_{t}^{0,1} \mathbf{1}_{\left\{t<\tau_{1}\right\}}+Y_{t}^{1}\left(\tau_{1}\right) \mathbf{1}_{\left\{\tau_{1} \leq t<\tau_{2}\right\}}+B^{3}\left(\tau_{1}, \tau_{2}\right) \mathbf{1}_{\left\{\tau_{1}<\tau_{2} \leq t\right\}}\right), \\
Z_{t}= & \mathbf{1}_{\left\{\tau_{2}<\tau_{1}\right\}}\left(Z_{t}^{0,2} \mathbf{1}_{\left\{t<\tau_{2}\right\}}+Z_{t}^{2}\left(\tau_{2}\right) \mathbf{1}_{\left\{\tau_{2} \leq t<\tau_{1}\right\}}\right) \\
& \quad+\mathbf{1}_{\left\{\tau_{1}<\tau_{2}\right\}}\left(Z_{t}^{0,1} \mathbf{1}_{\left\{t<\tau_{1}\right\}}+Z_{t}^{1}\left(\tau_{1}\right) \mathbf{1}_{\left\{\tau_{1} \leq t<\tau_{2}\right\}}\right), \\
U_{t}^{1}= & \mathbf{1}_{\left\{\tau_{2} \leq t<\tau_{1}\right\}}\left(B^{3}\left(t, \tau_{2}\right)-Y_{t}^{2}\left(\tau_{2}\right)\right)+\mathbf{1}_{\left\{t<\tau_{1}<\tau_{2}\right\}}\left(Y_{t}^{1}(t)-Y_{t}^{0,1}\right), \\
U_{t}^{2}= & \mathbf{1}_{\left\{t<\tau_{2}<\tau_{1}\right\}}\left(Y_{t}^{2}(t)-Y_{t}^{0,2}\right)+\mathbf{1}_{\left\{\tau_{1} \leq t<\tau_{2}\right\}}\left(B^{3}\left(\tau_{1}, t\right)-Y_{t}^{1}\left(\tau_{1}\right)\right) .
\end{aligned}\right.
$$

For more details on this formulation we refer to Appendix A.2. The latter exposes the main reasoning behind the proof, which is similar to the one considered in Kharroubi and Lim (2014). The above decomposition will play a key role in the numerical derivation of the indifference price $p^{*}$ as we will see in the next section.

\section{$3 \quad$ Numerical analysis}

3.1 Data source. We consider two real-world portfolios related to death guarantees. These are portfolios of males holding pure death benefit guarantees distributed over ages as in Figure 1. In the abscissa, we reported the percentage of individuals in the portfolio for each age between 0 and 120. The coordinates represent these ages. Here, the size of the portfolios is omitted may be specified later. Later on, we assess the effect of the size both on the representation of the portfolio as well as on the indifference price.

Notice that the composition of the two portfolios is different in the sense that the first portfolio is composed of individuals relatively young, with an average age of 44.7. The second 
is mainly concentrated on older ages with an average policyholder age of 64.1. For these portfolios, almost all individuals are aged over 20 . We also consider an age dependent intensity

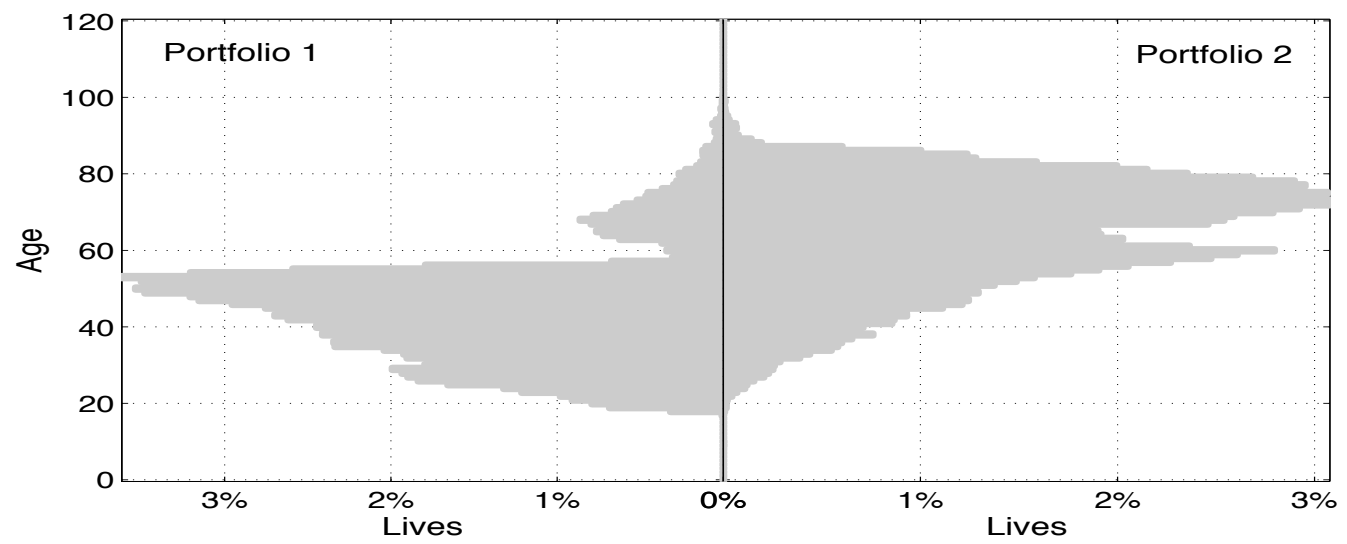

Figure 1: Distribution of individuals over ages in the portfolios.

displayed in Figure 2. This mortality curve corresponds to the French mortality table TPRV90. Initially, this table gives the one-year death probability at the age level. Here, we formulated the TPRV90 in terms of the intensity. The latter, in Figure 2, is constant for each age and gives the likelihood of the death occurrence of each individual. It is to be incorporated in the exponential model presented above.

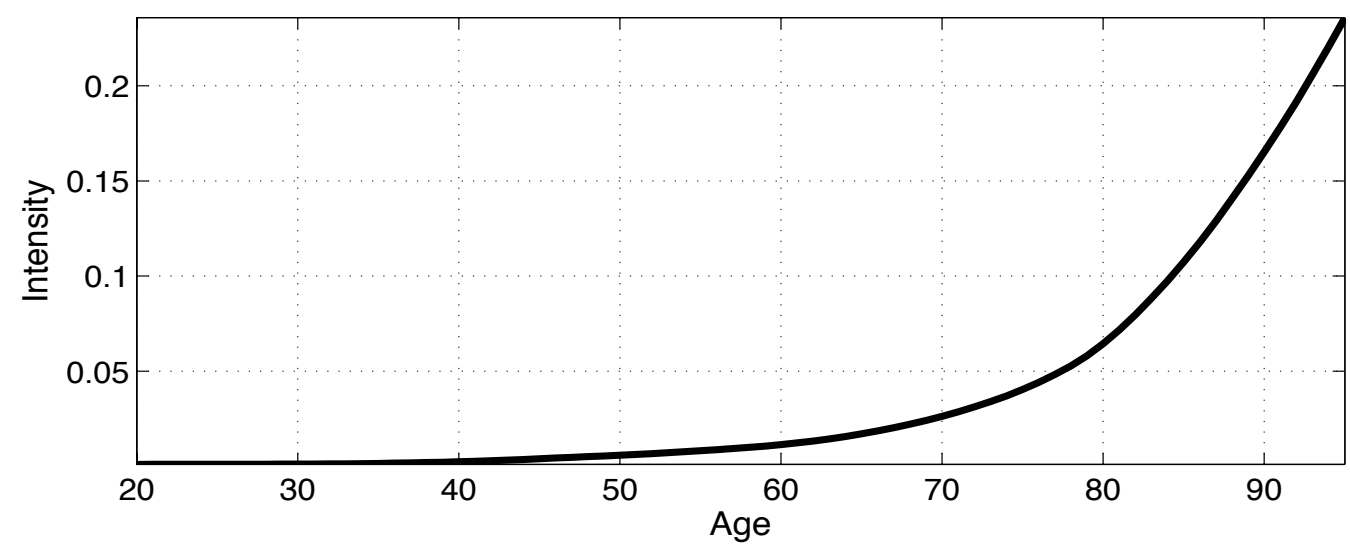

Figure 2: Mortality intensity per age.

3.2 Simulating death times. As in Section 1, we model the dependence structure of death times by an FGM copula. For the sake of applicability, we consider a special form of the general FGM copula in (1.1) to model this dependency. More precisely, we let the function

$$
C_{\theta}\left(u_{1}, \cdots, u_{n}\right)=\prod_{i=1}^{n} u_{i}\left(1+\theta \prod_{j=1}^{n}\left(1-u_{j}\right)\right)
$$

be the copula linking the marginals of the lifetimes such that $\theta \in[-1,1]$, see Genest et al. 
(2011). Using similar calculation in Subsection 1.2, we can easily see that

$$
\begin{aligned}
\frac{\partial}{\partial u_{k}} C_{\theta}\left(u_{1}, \ldots, u_{n}\right) & =\prod_{i=1, i \neq k}^{n} u_{i}\left(1+\theta\left(1-2 u_{k}\right) \prod_{i=1, i \neq k}^{n}\left(1-u_{i}\right)\right) \\
& =C_{\theta\left(1-2 u_{k}\right)}\left(u_{1}, \ldots, u_{k-1}, u_{k+1}, \ldots, u_{n}\right)
\end{aligned}
$$

where $C_{\theta\left(1-2 u_{k}\right)}$ is an FGM copula with parameter $\theta\left(1-2 u_{k}\right) \in[-1,1]$. As a consequence, sampling from $C_{\theta}$ is reduced to sampling from $C_{\theta\left(1-2 u_{k}\right)}$. To this end, the conditional distribution method can be used. More precisely, following Remillard (2013, Section 8.7.12), we can draw a vector $\left(v_{1}, \ldots, v_{n}\right) \sim \operatorname{Unif}([0,1])^{n}$ and set $u_{1}=v_{1}, \ldots, u_{n-1}=v_{n-1}$, and $u_{n}=2 v_{n} /\left(1+w+\sqrt{(1+w)^{2}-4 w v_{n}}\right)$, with $w=\theta \prod_{i=1}^{n-1}\left(1-2 v_{i}\right)$, then $\left(u_{1}, \ldots, u_{n}\right) \sim C_{\theta}$.
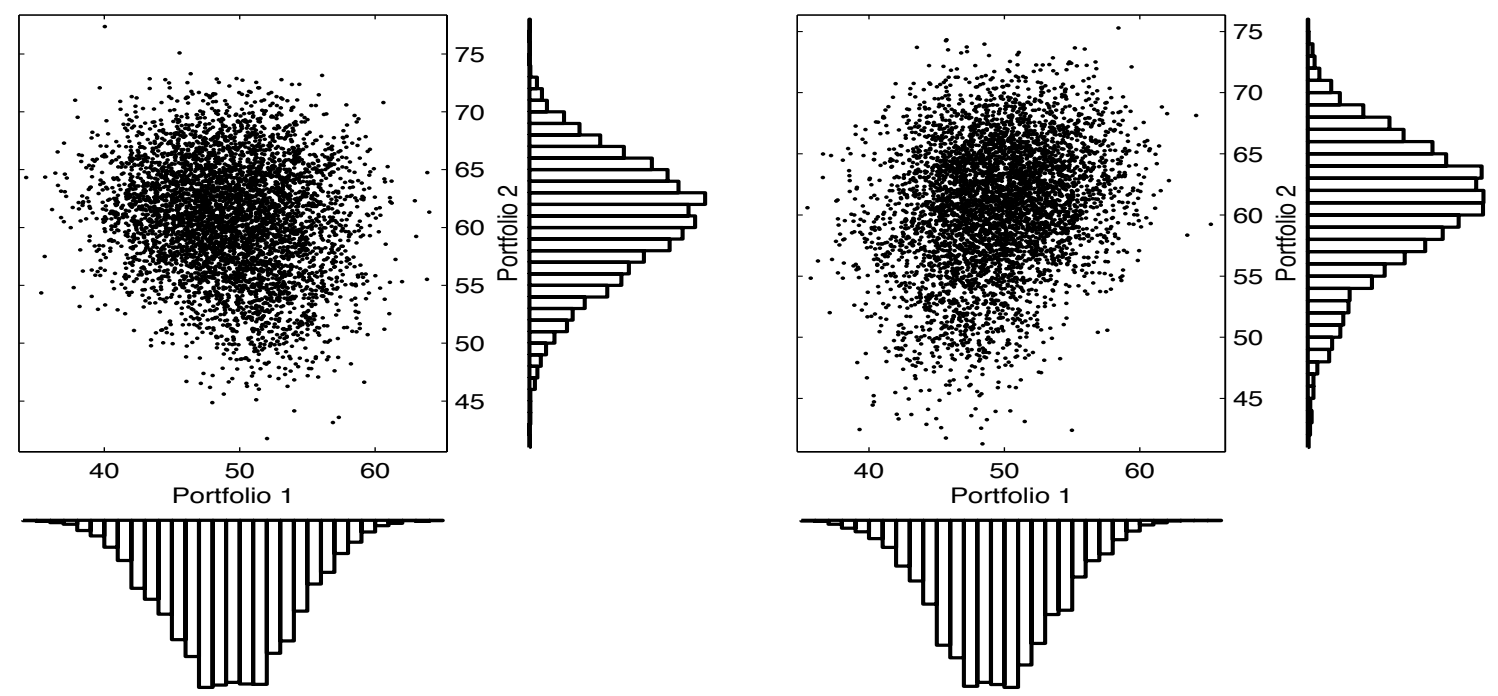

Figure 3: Correlation and histograms of the discounted pay-offs of the two portfolios with similar sizes of 100 individuals and homogeneous guarantees, i.e. $c_{i}=\tilde{c}_{i}=1$.

Figure 3 shows a 5000 samples of the aggregate exposure to two portfolios of $n=m=100$ individuals distributed over ages as displayed in Figure 1. Each individual is holding a term contract paying out 1 if she dies before the maturity term $T=10$. The scatter plots represent the distribution (right and bottom histograms) of the aggregate exposure for each portfolio. We used the copula function in (3.1) with a parameter $\theta=-0.7$ (left) and $\theta=0.7$ (right) respectively to draw the death times of the individuals. More precisely, we used the copula with the fixed parameter $\theta$ to simultaneously draw the remaining lifetimes of the 200 individuals, then split the lifetimes to form the two portfolios. In Figure 3, the values $\theta=0.7$ and $\theta=-0.7$ generate correlated (aggregated) exposures of the same corresponding signs. Indeed, for the case $\theta=-0.7$ the two portfolios are negatively correlated with a linear correlation being equal to $-25 \%$. In general, the linear correlation induced by an FGM copula with parameter $\theta$ is $\theta / 3$. In other words, using the copula in (3.1), we can expect a linear correlation between the two portfolios at most ranging from $-33 \%$ to $+33 \%$. This is in tune with empirical findings 
and common practical assumptions.

3.3 Representative portfolio. We consider two model points to represent two portfolios with a given correlation. We follow the procedure presented in Subsection 2.1. Table 1 gives the characteristics of the initial portfolios. Formally, each portfolio, denoted respectively $P 1$ and $P 2$, is characterized by its variance, expected discounted cash-flows as well as the correlation with the second portfolio. These specific characteristics are given for simulated portfolios and reported (between brackets) for different sizes of the underlying population. We consider, respectively, a small portfolio of 500 lives and a (relatively) big portfolio of 5000 lives, and thus for maturities $T=10$ and $T=15$ of the contracts. The first panel corresponds to two portfolios with 500 lives each distributed over ages respectively as in Figure 1. The two other panels correspond respectively to portfolios with 5000 lives for the second, and $n=5000$ and $m=500$ of the third. Simulations of these portfolios are drawn in the same manner as above with copula structure in Equation (3.1). Here, we consider respectively a negative, positive and zero correlation. For each case, in Table 1, we report the initial characteristics of the portfolios as well as those of the representative agents. First, we should note that a sample of death times is drawn once for each case. For example, for $n=m=500$ and positive correlation, we the $n+m$ simulated deaths are used for both maturities $T=10$ and 15. Therefore, we can remark that the correlation between $\mathrm{P} 1$ and $\mathrm{P} 2$ increases with maturity. The same conclusion arises for nil and negative correlation. This is related to the aging phenomena in the sense. Although the two portfolios are centered around different age tranches, the increase of maturity will increase the number of the deceased individuals in the first portfolio P1 as well as in P2. This shall increase the number of joint realization of deaths in P1 and P2. Consequently, a linear correlation initially calculated on a small maturity increases when going from $T=10$ to $T=15$.

As soon as the performance of the representation is concerned, we notice that the portfolios' characteristics are well described by the model points. In particular, the negative correlation between the initial portfolios is well replicated. The representative agents also replicate the independence case. This particular feature is ensured given the criterion used to characterize the agents. Generally, the representative agents succeed to represent $90 \%$ to $95 \%$ of the initial portfolios risk profiles (variance and expected cash-flows) as well as the correlation between these portfolios, see Table 1. Notice that the size of the portfolios or the maturity of the contracts do not impact this performance.

3.4 Indifference price. The indifference price derivation is given in terms of the unique solutions of the BSDEs in Proposition 2 and Proposition 3. Therefore, we need to simulate the BSDEs to get the values $y_{0}$ and $Y_{0}$ characterizing the price $p^{*}$ in Corollary 1 . To do so, we introduce a discrete-time approximation of the solutions $(Y, Z, U)$ and $(y, z, u)$ following similar ideas used in Kharroubi and Lim $(2011,2012)$.

We consider a discretization grid $0=t_{0}<t_{1}<\cdots<t_{k}=T$ of $[0, T]$ and for $u \in \mathbb{R}$ we let $\rho(u)=\max \left\{t_{i}, i=0, \cdots, k \mid t_{i} \leq u\right\}$ be the largest element of the grid smaller than $u$. Moreover, we use the notation $\Delta W_{t_{i}}$ to denote the increment of $W$ between $t_{i-1}$ and $t_{i}$ for $i=0, \cdots, k$ and $(Y, Z, U)$ will present the discretized solution of the initial BSDE. For convenience, we suppose that the discretization grid has constant mesh denoted $\Delta_{k}$ and 
Table 1: Analysis of the representative model for different sizes of the portfolios and times horizons of the contract

\begin{tabular}{|c|c|c|c|c|c|c|c|c|c|c|}
\hline & & \multicolumn{3}{|c|}{$n=m=500$} & \multicolumn{3}{|c|}{$n=m=5000$} & \multicolumn{3}{|c|}{$n=5000 ; m=500$} \\
\hline & & $\mathrm{P} 1$ & $\mathrm{P} 2$ & $\mathrm{P} 1+\mathrm{P} 2$ & $\mathrm{P} 1$ & $\mathrm{P} 2$ & $\mathrm{P} 1+\mathrm{P} 2$ & $\mathrm{P} 1$ & $\mathrm{P} 2$ & $\mathrm{P} 1+\mathrm{P} 2$ \\
\hline \multirow{8}{*}{ 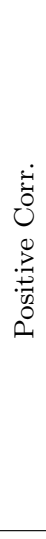 } & & \multicolumn{3}{|c|}{$\mathrm{T}=10$} & \multicolumn{3}{|c|}{$\mathrm{T}=10$} & \multicolumn{3}{|c|}{$\mathrm{T}=10$} \\
\hline & Var. & $\begin{array}{c}282.36 \\
(308.01)\end{array}$ & $\begin{array}{c}99.59 \\
(105.83)\end{array}$ & $\begin{array}{c}436.72 \\
(477.86)\end{array}$ & $\begin{array}{c}1063.28 \\
(1069.35)\end{array}$ & $\begin{array}{c}1069.35 \\
(1006.15)\end{array}$ & $\begin{array}{c}2305.81 \\
(2244.11)\end{array}$ & $\begin{array}{c}17941.91 \\
(18737.95)\end{array}$ & $\begin{array}{c}83.23 \\
(70.55)\end{array}$ & $\begin{array}{c}18333.33 \\
(19074.52)\end{array}$ \\
\hline & Mean & $\begin{array}{c}186.71 \\
(193.55)\end{array}$ & $\begin{array}{c}235.33 \\
(241.02)\end{array}$ & $\begin{array}{c}422.04 \\
(434.57)\end{array}$ & $\begin{array}{c}1918.12 \\
(1946.95)\end{array}$ & $\begin{array}{c}2395.57 \\
(2426.75)\end{array}$ & $\begin{array}{c}4313.69 \\
(4373.70)\end{array}$ & $\begin{array}{c}1888.65 \\
(1948.00)\end{array}$ & $\begin{array}{c}235.66 \\
(240.96)\end{array}$ & $\begin{array}{c}2124.31 \\
(2188.96)\end{array}$ \\
\hline & Corr. & \multicolumn{3}{|c|}{$\begin{array}{c}16.32 \% \\
(17.72 \%)\end{array}$} & \multicolumn{3}{|c|}{$\begin{array}{c}13.24 \% \\
(12.11 \%)\end{array}$} & \multicolumn{3}{|c|}{$\begin{array}{c}12.60 \% \\
(11.56 \%)\end{array}$} \\
\hline & & \multicolumn{3}{|c|}{$\mathrm{T}=15$} & \multicolumn{3}{|c|}{$\mathrm{T}=15$} & \multicolumn{3}{|c|}{$\mathrm{T}=15$} \\
\hline & Var. & $\begin{array}{c}99.35 \\
(110.21)\end{array}$ & $\begin{array}{c}271.92 \\
(261.38)\end{array}$ & $\begin{array}{c}474.58 \\
(465.98)\end{array}$ & $\begin{array}{c}1005.65 \\
(1030.33)\end{array}$ & $\begin{array}{c}928.46 \\
(910.04)\end{array}$ & $\begin{array}{c}2153.38 \\
(2159.77)\end{array}$ & $\begin{array}{c}1490.81 \\
(1509.70)\end{array}$ & $\begin{array}{c}229.40 \\
(352.73)\end{array}$ & $\begin{array}{c}2318.76 \\
(2301.83)\end{array}$ \\
\hline & Mean & 242.19 & 250.19 & 492.38 & 2479.15 & 2992.61 & 5471.76 & 2471.40 & 288.63 & 1720.21 \\
\hline & Corr. & $\begin{array}{r}31 \\
(27\end{array}$ & $\%$ & & $\begin{array}{r}19 . \\
(18 .\end{array}$ & $\%$ & & $\begin{array}{r}54.2 \\
(40.1\end{array}$ & 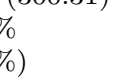 & \\
\hline \multirow{8}{*}{ 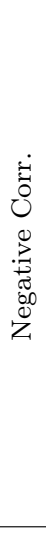 } & & \multicolumn{3}{|c|}{$\mathrm{T}=10$} & \multicolumn{3}{|c|}{$\mathrm{T}=10$} & \multicolumn{3}{|c|}{$\mathrm{T}=10$} \\
\hline & Var. & $\begin{array}{c}304.11 \\
(300.97)\end{array}$ & $\begin{array}{c}136.12 \\
(190.47)\end{array}$ & $\begin{array}{c}352.98 \\
(432.73)\end{array}$ & $\begin{array}{l}1067.69 \\
(827.40)\end{array}$ & $\begin{array}{l}1063.90 \\
(947.15)\end{array}$ & $\begin{array}{c}1959.27 \\
(1668.65)\end{array}$ & $\begin{array}{c}17941.91 \\
(17935.08)\end{array}$ & $\begin{array}{c}83.23 \\
(70.02)\end{array}$ & $\begin{array}{l}17841.0447 \\
(17828.74)\end{array}$ \\
\hline & Mean & $\begin{array}{c}186.83 \\
(193.89)\end{array}$ & $\begin{array}{c}234.37 \\
(241.01)\end{array}$ & $\begin{array}{c}421.20 \\
(434.90)\end{array}$ & $\begin{array}{c}1918.05 \\
(1947.88)\end{array}$ & $\begin{array}{c}2395.70 \\
(2428.12)\end{array}$ & $\begin{array}{c}4313.75 \\
(4376.00)\end{array}$ & $\begin{array}{c}1889.04 \\
(1948.00)\end{array}$ & $\begin{array}{l}235.946 \\
(240.96)\end{array}$ & $\begin{array}{l}2124.986 \\
(2188.96)\end{array}$ \\
\hline & Corr. & \multicolumn{3}{|c|}{$\begin{array}{c}-21.43 \% \\
(-12.26 \%)\end{array}$} & \multicolumn{3}{|l|}{$\begin{array}{l}-19.17 \% \\
(-16.82) \%\end{array}$} & \multicolumn{2}{|c|}{$\begin{array}{l}-12.61 \% \\
(-12.17 \%)\end{array}$} & \\
\hline & & \multicolumn{3}{|c|}{$\mathrm{T}=15$} & \multicolumn{3}{|c|}{$\mathrm{T}=15$} & \multicolumn{3}{|c|}{$\mathrm{T}=15$} \\
\hline & Var. & $\begin{array}{c}115.79 \\
(103.22)\end{array}$ & $\begin{array}{c}322.46 \\
(324.06)\end{array}$ & $\begin{array}{c}410.25 \\
(393.71)\end{array}$ & $\begin{array}{c}1036.12 \\
(1019.29)\end{array}$ & $\begin{array}{c}907.93 \\
(913.59)\end{array}$ & $\begin{array}{c}1885.75 \\
(1869.54)\end{array}$ & $\begin{array}{c}1462.72 \\
(1442.12)\end{array}$ & $\begin{array}{c}228.77 \\
(245.45)\end{array}$ & $\begin{array}{c}1653.91 \\
(1641.08)\end{array}$ \\
\hline & Mean & 242.11 & 288.76 & 530.87 & 2478.87 & 2992.31 & 5471.18 & 2472.85 & 289.32 & 2762.17 \\
\hline & Corr. & \multicolumn{3}{|c|}{$\begin{array}{l}-7.24 \% \\
(-9.18 \%)\end{array}$} & \multicolumn{2}{|c|}{$\begin{array}{c}-9.31 \% \\
(-11.02 \%)\end{array}$} & $(5534.77)$ & \multicolumn{2}{|c|}{$\begin{array}{l}-1.31 \% \\
(-1.09 \%)\end{array}$} & $(2811.72)$ \\
\hline \multirow{8}{*}{ 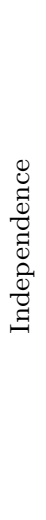 } & & \multicolumn{3}{|c|}{$\mathrm{T}=10$} & \multicolumn{3}{|c|}{$\mathrm{T}=10$} & \multicolumn{3}{|c|}{$\mathrm{T}=10$} \\
\hline & Var. & $\begin{array}{l}104.96 \\
(95.94)\end{array}$ & $\begin{array}{c}106.63 \\
(104.83)\end{array}$ & $\begin{array}{c}213.13 \\
(199.55)\end{array}$ & $\begin{array}{l}1067.69 \\
(833.65)\end{array}$ & $\begin{array}{l}1063.90 \\
(933.31)\end{array}$ & $\begin{array}{c}2165.82 \\
(1811.46)\end{array}$ & $\begin{array}{l}1063.54 \\
(104.82)\end{array}$ & $\begin{array}{c}104.81 \\
(103.01)\end{array}$ & $\begin{array}{l}1179.37 \\
(989.33)\end{array}$ \\
\hline & Mean & $\begin{array}{c}188.79 \\
(193.80)\end{array}$ & $\begin{array}{c}235.34 \\
(241.05)\end{array}$ & $\begin{array}{c}424.13 \\
(434.85)\end{array}$ & $\begin{array}{c}1918.10 \\
(1947.88)\end{array}$ & $\begin{array}{c}2396.03 \\
(2428.12)\end{array}$ & $\begin{array}{c}4314.13 \\
(4376.00)\end{array}$ & $\begin{array}{c}1917.81 \\
(1947.80)\end{array}$ & $\begin{array}{l}235.492 \\
(240.99)\end{array}$ & $\begin{array}{l}2153.302 \\
(2188.79)\end{array}$ \\
\hline & Corr. & \multicolumn{3}{|c|}{$\begin{array}{c}0.73 \% \\
(1.09 \%)\end{array}$} & $\begin{array}{l}0.2 \\
(0.1 \\
\end{array}$ & & & $\begin{array}{r}1.6 \\
(2.9\end{array}$ & & \\
\hline & & & $\mathrm{T}=15$ & & & $\mathrm{~T}=15$ & & & $\mathrm{~T}=15$ & \\
\hline & Var. & $\begin{array}{c}100.62 \\
(103.04)\end{array}$ & $\begin{array}{c}90.82 \\
(94.35)\end{array}$ & $\begin{array}{l}190.90 \\
(197.64)\end{array}$ & $\begin{array}{c}1036.12 \\
(1018.05)\end{array}$ & $\begin{array}{l}907.93 \\
(916.06)\end{array}$ & $\begin{array}{c}1935.09 \\
(1925.24)\end{array}$ & $\begin{array}{c}986.42 \\
(1051.09)\end{array}$ & $\begin{array}{c}90.5 \\
(98.09)\end{array}$ & $\begin{array}{c}1082.8 \\
(1164.90)\end{array}$ \\
\hline & Mean & $\begin{array}{c}242.13 \\
(250.06)\end{array}$ & $\begin{array}{c}292.14 \\
(300.37)\end{array}$ & $\begin{array}{c}534.27 \\
(550.43)\end{array}$ & $\begin{array}{c}2478.42 \\
(2510.79)\end{array}$ & $\begin{array}{c}2992.71 \\
(3023.98)\end{array}$ & $\begin{array}{c}5471.13 \\
(5534.77)\end{array}$ & $\begin{array}{c}2479.34 \\
(2510.78)\end{array}$ & $\begin{array}{c}292.15 \\
(300.54)\end{array}$ & $\begin{array}{c}2771.49 \\
(2811.32)\end{array}$ \\
\hline & Corr. & $\begin{array}{r}0 . \\
(0 .\end{array}$ & $\begin{array}{l}\% \\
\%)\end{array}$ & & 0.2 & $\begin{array}{l}\% \\
\%\end{array}$ & & $\begin{array}{r}0.9 \\
(2.4\end{array}$ & $\begin{array}{l}\text { (200.07 } \\
0 \\
0)\end{array}$ & \\
\hline
\end{tabular}


consider a classical backward implicit schemes on $\rho$ associated with the system of BSDEs. Before proceeding to the numerical implementation note that we only need to simulate (2.6) or (2.7) as the price $p^{*}$ is given in terms of $Y_{0}$ which coincides with $Y_{0}^{0,1}$ and $Y_{0}^{0,2}$. Here, we develop the simulation scheme for the BSDE in (2.6) as follows:

$$
\begin{cases}Y_{T}^{0,1} & =B^{0} \\ Z_{t_{i-1}}^{0,1} & =\frac{1}{\Delta_{k}} \mathbb{E}\left[Y_{t_{i}}^{0,1} \Delta W_{t_{i}} \mid \mathcal{F}_{t_{i-1}}\right] \\ Y_{t_{i-1}}^{0,1} & =f^{0}\left(t_{i-1}, Y_{t_{i-1}}^{0,1}, Z_{t_{i-1}}^{0,1}, Y_{t_{i-1}}^{1}\left(t_{i-1}\right)-Y_{t_{i-1}}^{0,1}, 0\right) \Delta_{k}+\mathbb{E}\left[Y_{t_{i}}^{0,1} \mid \mathcal{F}_{t_{i-1}}\right]\end{cases}
$$

where $Y^{1}$ is given by the following Euler scheme:

$$
\left\{\begin{aligned}
Y_{T}^{1}\left(\rho\left(\theta_{1}\right)\right)= & B^{1}\left(\rho\left(\theta_{1}\right)\right) \\
Z_{t_{i-1}}^{1} & =\frac{1}{\Delta_{k}} \mathbb{E}\left[Y_{t_{i}}^{1}\left(\rho\left(\theta_{1}\right)\right) \Delta W_{t_{i}} \mid \mathcal{F}_{t_{i-1}}\right] \\
Y_{t_{i-1}}^{1}\left(\rho\left(\theta_{1}\right)\right)= & f^{1}\left(t_{i-1}, Y_{t_{i-1}}^{1}\left(\rho\left(\theta_{1}\right)\right), Z_{t_{i-1}}^{1}\left(\rho\left(\theta_{1}\right)\right), B^{3}\left(\rho\left(\theta_{1}\right), t_{i-1}\right)-Y_{t_{i-1}}^{1}\left(\rho\left(\theta_{i}\right)\right), \rho\left(\theta_{1}\right)\right) \Delta_{k} \\
& \quad+\mathbb{E}\left[Y_{t_{i}}^{1}\left(\rho\left(\theta_{1}\right)\right) \mid \mathcal{F}_{t_{i-1}}\right]
\end{aligned}\right.
$$

with $\theta_{1} \geq 0$. Similarly, the solution $y_{0}$ needed to determine the value function $V$ can be derived using a Euler scheme, see Chevalier et al. (2014) for more details. In order to simulate the discretized BSDE above, we compute the conditional expectations involved using a the regression method in Gobet et al. (2005) based on $L^{2}$-projections on finite bases. This consists on a regression over a predefined polynomial basis of degree $R$. The simulation developed in the sequel uses the following set of parameters $k=120, R=30$ and Laguerre polynomials to approximate the conditional expectation.

For the numerical illustrations, we consider two representative agents with intensity $\lambda$ and $\tilde{\lambda}$ and guaranteed amount $c$ and $\tilde{c}$ and correlation structure of FGM form parametrized by the coefficient $\theta$. The first individual represents the initial exposure and the second is the acquired portfolio. Recall that we mainly focus on the death benefit policies. As noted before $c$ and $\tilde{c}$ are the expected payment in case the individual dies before maturity $T$. In the sequel we consider the impact of various parameters on the indifference price. To do so, we consider a flat interest rate curve $r$ and homogeneous and deterministic equity return $\mu$ and volatility $\sigma$. We shall give the following numerical values to these parameters:

$$
r=0.02, \quad \mu=0.15, \quad \sigma=0.3 .
$$

Regarding the parameter $\alpha$ that measures the absolute risk aversion of the insurer, Young (2003) noted that a typical of value for a well-capitalized insurer is very small. Thus, we let $\alpha=0.3$. Henceforth, the numerical study is divided in three parts. First, we consider a pure endowment guarantee and describe the dependence of the price with respect to the portfolios parameters and risk profile: the contracts maturity, the intensity of the representative agents, the guaranteed amount. Next, we investigate the impact of the dependence parameter $\theta$. Finally, we consider the price sensitivity with respect to the financial market parameters. For the correlation impact, we are considering the values $\theta=0$ (for independence), $\theta=-0.3$ (for negative correlation) and $\theta=0.3$. The indifference price $p^{*}$ will be compared to the classical 
actuarial price given as $p=c \mathbb{E}\left[e^{-r \tau \wedge T} \mathbf{1}_{\{\tau<T\}}\right]=c g(\lambda)$, where $g$ is given in Assumption 2 for death benefit contracts. In view of the parameters value above and the intensity depicted in Figure 2. Table 2 reports the actuarial price for different contract maturities and individual mortality intensity (with $c=1$ ).

Table 2: Actuarial price for death benefits contracts

\begin{tabular}{llll}
\hline & $T=5$ & $T=10$ & $T=15$ \\
\cline { 2 - 4 }$\lambda=\tilde{\lambda}=0.01$ & 0.0464 & 0.0864 & 0.1208 \\
$\lambda=\tilde{\lambda}=0.03$ & 0.1327 & 0.2361 & 0.3166 \\
$\lambda=\tilde{\lambda}=0.05$ & 0.2109 & 0.3596 & 0.4643 \\
\hline
\end{tabular}

First, we implement the procedure to assess the impact of the initial size of the portfolio as well as the mortality intensity. In Table 3 we reported the results for $c=100,5000$ and $\lambda=0.01,0.05$ for fixed $\tilde{c}=1$. We remark that the price for an additional contract charged by the insurer is increasing with $\lambda$ as the guaranteed amount is likely to be paid during the maturity of the contract. Moreover, we remark that the price does not depend on the initial size c. In fact, the indifference pricing does account for the pooling effect which a already known property. In general, the prices reported in Table 3 are greater that the classical actuarial prices reported in Table 2. There is, however, an exception for the case when the dependence coefficient $\theta$ is negative. The price is such a case in less that the actuarial price. This may be due to the preference of the insurer incorporated in $p^{*}$ which makes the latter prefer the risk that reduces its initial exposure and charge less fees compared to the positive dependency case as well. The table also shows that the price $p^{*}$ is increasing with maturity.

Table 3: Indifference price for different contract maturities, mortality intensities and correlation coefficient.

\begin{tabular}{lccc}
\hline & $T=5$ & $T=10$ & $T=15$ \\
\hline \hline & \multicolumn{3}{c}{$\theta=+0.3$} \\
\cline { 2 - 4 }$\lambda=\tilde{\lambda}=0.01$ & 0.0678 & 0.1393 & 0.2070 \\
$\lambda=\tilde{\lambda}=0.03$ & 0.1739 & 0.2823 & 0.3917 \\
$\lambda=\tilde{\lambda}=0.05$ & 0.3393 & 0.4691 & 0.5635 \\
\hline \hline & \multicolumn{3}{c}{$\theta=0$} \\
\cline { 2 - 4 }$\lambda=\tilde{\lambda}=0.01$ & 0.0600 & 0.1222 & 0.1848 \\
$\lambda=\tilde{\lambda}=0.03$ & 0.1499 & 0.2392 & 0.3292 \\
$\lambda=\tilde{\lambda}=0.05$ & 0.2993 & 0.4191 & 0.5235 \\
\hline \hline & \multicolumn{3}{c}{$\theta=-0.3$} \\
$\lambda=\tilde{\lambda}=0.01$ & 0.0513 & 0.1025 & 0.1593 \\
$\lambda=\tilde{\lambda}=0.03$ & 0.1281 & 0.2007 & 0.2838 \\
$\lambda=\tilde{\lambda}=0.05$ & 0.1910 & 0.2936 & 0.3897 \\
\hline
\end{tabular}

In Table 4 , we reported the quantity $p^{*} / \tilde{c}$ for $\tilde{c}=1,100,1000$ and fixed $c=1000$, which 
can be interpreted as the indifference price per unit. We should mention that this corresponds the representative guaranteed quantity which should be smaller that the aggregate sums at risk in the initial portfolio. However, we note that $p^{*} / \tilde{c}$ is increasing in $\tilde{c}$. In other words, the indifference price for additional exposures to mortality risk (even if it is negatively correlated to its initial exposure), is higher when it increases. This is a typical result related to the indifference pricing with exponential utilities. The so-called result for the indifference price of $k X$ where $X$ is a single-event contingent claim was proven by Becherer (2003, Corollary 3.3) in a general semi-martingale incomplete market. Similar effect of the size on the prices of pure endowment contracts is showed by Ludkovski and Young (2008).

Table 4: Marginal indifference price as a function of the guaranteed amount $\tilde{c}$ of the second represenatative agent with $c=1000$.

\begin{tabular}{lccc}
\hline & $\tilde{c}=1$ & $\tilde{c}=100$ & $\tilde{c}=1000$ \\
\cline { 2 - 4 }$\theta=+0.3$ & 0.2823 & 0.2918 & 0.3070 \\
$\theta=0$ & 0.2392 & 0.2473 & 0.2601 \\
$\theta=-0.3$ & 0.2007 & 0.2074 & 0.2182 \\
\hline
\end{tabular}

\section{Conclusion}

In this paper, we investigated the problem of pricing term life insurance contracts using the indifference pricing approach. We considered an insurer with an initial exposure that wants to sell new contracts or buy a whole run-off portfolio. We focused on the case where the lifetimes of the individuals are correlated and suppose that the latter is captured by an FGM copula.

The indifference pricing methodology relies on the solutions of BSDEs with jumps. The latter, is generally hard to simulate numerically when the as it involves high dimensional BSDEs. To overcome this issue, we propose to substitute the initial portfolios with two correlated representative agents that best capture their risk profile as well as some of their statistical features. Based on this new policyholders, we are then able to implement the pricing methodology and simulate the related BSDEs.

The representative methodology as well as the price of death benefit portfolios are numerically analyzed. Among others, we investigate the impact of the contracts design, the dependence structure as well as the financial market parameters.

\section{References}

P. Barrieu, H. Bensusan, N. El Karoui, C. Hillairet, S. Loisel, C. Ravanelli, and Y. Salhi. Understanding, modelling and managing longevity risk: key issues and main challenges. Scandinavian actuarial journal, 2012(3):203-231, 2012.

E. Bayraktar, M.A. Milevsky, S.D. Promislow, and V.R. Young. Valuation of mortality risk via the instantaneous sharpe ratio: applications to life annuities. Journal of Economic Dynamics and Control, 33(3):676-691, 2009.

D. Becherer. Rational hedging and valuation of integrated risks under constant absolute risk aversion. Insurance: Mathematics and economics, 33(1):1-28, 2003. 
A.J.G. Cairns, D. Blake, and K. Dowd. Pricing frameworks for securitization of mortality risk. In Proceedings of the 14th AFIR Colloquium, volume 1, pages 509-540, 2004.

L.R. Carter and R.D. Lee. Modeling and forecasting us sex differentials in mortality. International Journal of Forecasting, 8(3):393-411, 1992.

E. Chevalier, T. Lim, and R.R. Roméro. Indifference fee rate for variable annuities. 2014.

S. H Cox and Y. Lin. Natural hedging of life and annuity mortality risks. North American Actuarial Journal, 11(3):1-15, 2007.

Ł. Delong. No-good-deal, local mean-variance and ambiguity risk pricing and hedging for an insurance payment process. Astin Bulletin, 42(01):203-232, 2012.

N. El Karoui, M. Jeanblanc, and Y. Jiao. Density approach in modelling multi-defaults. preprint, hal-00870492, 2013.

C. Genest, J. Nešlehová, and N. Ben Ghorbal. Estimators based on kendall's tau in multivariate copula models. Australian $\&$ New Zealand Journal of Statistics, 53(2):157-177, 2011.

E. Gobet, J.-P. Lemor, and X. Warin. A regression-based monte carlo method to solve backward stochastic differential equations. The Annals of Applied Probability, 15(3):2172-2202, 2005.

P.-Q. Goffard and X. Guerrault. Is it optimal to group policyholders by age, gender, and seniority for bel computations based on model points? European Actuarial Journal, pages 1-16, 2015.

S.D. Hodges and A. Neuberger. Optimal replication of contingent claims under transaction costs. Review of futures markets, 8(2):222-239, 1989.

Y. Hu, P. Imkeller, and M. Muller. Utility maximization in incomplete markets. Annals of Probability, 15(3):1691-1712, 2005.

P. Jaworski, E. Durante, W.K. Hardle, and T. Rychlik. Copula theory and its applications. Springer, 2010.

I. Kharroubi and T. Lim. A decomposition approach for the discrete-time approximation of fbsdes with a jump i: the lipschitz case. arXiv preprint arXiv:1103.3029, 2011.

I. Kharroubi and T. Lim. A decomposition approach for the discrete-time approximation of bsdes with a jump ii: the quadratic case. arXiv preprint arXiv:1211.6231, 2012.

I. Kharroubi and T. Lim. Progressive enlargement of filtrations and backward stochastic differential equations with jumps. Journal of Theoretical Probability, 27(3):683-724, 2014.

I. Kharroubi, T. Lim, and A. Ngoupeyou. Mean-variance hedging on uncertain time horizon in a market with a jump. Applied Mathematics \& Optimization, 68(3):413-444, 2013.

M. Ludkovski and V.R. Young. Indifference pricing of pure endowments and life annuities under stochastic hazard and interest rates. Insurance: Mathematics and Economics, 42(1):14-30, 2008.

E. Marceau and P. Gaillardetz. On life insurance reserves in a stochastic mortality and interest rates environment. Insurance: Mathematics and Economics, 25(3):261-280, 1999.

M.A. Milevsky and S.D. Promislow. Mortality derivatives and the option to annuitise. Insurance: Mathematics and Economics, 29(3):299-318, 2001.

B. Remillard. Statistical Methods for Financial Engineering. CRC Press, 2013.

Virginia R Young. Equity-indexed life insurance: pricing and reserving using the principle of equivalent utility. North American Actuarial Journal, 7(1):68-86, 2003. 


\section{A Appendix}

A.1 Optimization problem with $n \geq 1$ policies. Suppose that the insurance company seeks to determine the optimal indifference price she applies for selling an extra contract. In the remainder of this section we will need the following notation. Let $\sigma=(\sigma(1), \cdots, \sigma(n))$ be a permutation of $\{1, \cdots, n\}$, and denote $\mathfrak{S}_{n}$ all such permutations. For all $i, j \in\{1, \cdots, n\}$, such that $i \leq j$, and $\sigma \in \mathfrak{S}_{n}$, we consider the death events $\left\{\tau_{i}, \cdots, \tau_{j}\right\}$ and we use the notation $\tau_{\sigma}(i, j)=\left\{\tau_{\sigma(i)}, \cdots, \tau_{\sigma(j)}\right\}, \bar{\tau}_{\sigma}^{i j}=\tau_{\sigma(i)} \vee \cdots \vee \tau_{\sigma(j)}$ and $\underline{\tau}_{\sigma}^{i j}=\tau_{\sigma(i)} \wedge \cdots \wedge \tau_{\sigma(j)}$. Note that for $i=j, \tau_{\sigma(i)}=\bar{\tau}_{\sigma}^{i i}=\underline{\tau}_{\sigma}^{i i}=\tau_{\sigma}(i, i)$ and for all $\sigma \in \mathfrak{S}_{n}$ we have $\bar{\tau}_{\sigma}^{1 n}=\bar{\tau}^{1 n}=\tau_{1} \vee \cdots \vee \tau_{n}$ and $\underline{\tau}_{\sigma}^{1 n}=\underline{\tau}^{1 n}=\tau_{1} \wedge \tau_{2} \cdots \wedge \tau_{n}$.

When the insurance company has sold $n$ contracts, the corresponding optimization of the expected value of the utility of its terminal wealth at time is given by:

$$
V:=\sup _{\pi \in \mathcal{A}_{\mathbb{G}^{n}}\left[0, T \wedge \bar{\tau}^{1 n}\right]} \mathbb{E}\left[U\left(X_{T \wedge \bar{\tau}^{1 n}}^{\pi}-\sum_{i=1}^{n} F_{T \wedge \tau_{i}}^{i}\right)\right] .
$$

In the case of an exponential utility, we note $B=\sum_{i=1}^{n} F_{T \wedge \tau_{i}}^{i}$ and the problem becomes:

$$
V=\sup _{\pi \in \mathcal{A}_{\mathbb{G}}\left[0, T \wedge \bar{\tau}^{1 n}\right]} \mathbb{E}\left[-\exp \left(-\alpha\left(X_{T \wedge \bar{\tau}^{1 n}}^{\pi}-B\right)\right)\right]
$$

where $B$ has the form

$$
B=B^{0} \mathbf{1}_{\left\{T<\underline{\tau}^{1 n}\right\}}+\sum_{\sigma \in \mathfrak{S}_{n}} \sum_{i=1}^{n-1} \mathbf{1}_{\left\{\bar{\tau}_{\sigma}^{1 i} \leq T<\underline{\tau}_{\sigma}^{i+1, n}\right\}} B_{\sigma}^{i}(\tau(1, i))+\mathbf{1}_{\left\{\bar{\tau}^{1 n} \leq T\right\}} B^{n}(\tau(1, n)),
$$

with $B^{0}=\sum_{j=1}^{n} F_{T}^{j}, B_{\sigma}^{i}=\sum_{j=1}^{i} F_{\tau_{\sigma(j)}}^{\sigma(j)}+\sum_{j=i+1}^{n} F_{T}^{\sigma(j)}$ and $B^{n}=\sum_{j=1}^{n} F_{\tau_{j}}^{j}$, where by convention $\sum_{j=1}^{0}=0$.

Proposition 4. Under the assumptions of Section 1:

1. The value function $\tilde{V}$ is given by $\tilde{V}(x)=-\exp \left(\alpha Y_{0}\right)$ where $Y_{0}$ is the initial value of the unique solution $(Y, Z, U)$ of the following BSDE

$$
Y_{t}=B+\int_{t \wedge \bar{\tau}^{1 n}}^{T \wedge \bar{\tau}^{1 n}} f\left(s, Y_{s}, Z_{s}, U_{s}\right) d s-\int_{t \wedge \bar{\tau}^{1 n}}^{T \wedge \bar{\tau}^{1 n}} Z_{s} d W_{s}-\int_{t \wedge \bar{\tau}^{1 n}}^{T \wedge \bar{\tau}^{1 n}} \sum_{i=1}^{n} U_{s}^{i} d H_{s}^{i}, \quad 0 \leq t \leq T,
$$

with $f(t, z, u)=\sum_{i=1}^{n}\left(1-H_{t}^{i}\right) \frac{e^{\alpha U_{t}^{i}-1}}{\alpha} \lambda_{t}^{i}-\frac{z\left(\mu_{t}-r\right)}{\sigma_{t}}-\frac{1}{2 \alpha} \frac{\left(\mu_{t}-r\right)^{2}}{\sigma_{t}^{2}}$.

There exists an optimal strategy $\hat{\pi}$ which satisfies

$$
\hat{\pi}_{t}=\frac{1}{\sigma_{t}}\left(Z_{t}+\frac{\mu_{t}-r}{\alpha \sigma_{t}}\right) .
$$

2. Moreover we suppose that

(a) $B \in \mathcal{G}_{\mathcal{T}}$, so $B=B^{0} \mathbf{1}_{\left\{T<\underline{\tau}^{1 n}\right\}}+\sum_{\sigma \in \mathfrak{S}_{n}} \sum_{i=1}^{n-1} \mathbf{1}_{\left\{\bar{\tau}_{\sigma}^{1 i} \leq T<\underline{\tau}_{\sigma}^{i+1, n}\right\}} B_{\sigma}^{i}(\tau(1, i))+\mathbf{1}_{\left\{\bar{\tau}_{\sigma}^{1 n} \leq T\right\}} B^{n}(\tau(1, n))$ 
(b) $f(t, y, z, u)=f^{0}(t, y, z, u) \mathbf{1}_{\left\{T<\underline{\tau}^{1 n}\right\}}+\sum_{\sigma \in \mathfrak{S}_{n}} \sum_{i=1}^{n-1} \mathbf{1}_{\left\{\bar{\tau}_{\sigma}^{1 i} \leq T<\underline{\tau}_{\sigma}^{i+1, n}\right\}} f_{\sigma}^{i}(t, y, z, u, \tau(1, i))+$

$$
\mathbf{1}_{\left\{\bar{\tau}^{1 n} \leq T\right\}} f^{n}(t, y, z, u, \tau(1, n)) .
$$

(c) Let $\sigma \in \mathfrak{S}_{n}$. For $1 \leq i \leq n-1$ the following BSDEs admit a solution

$$
\begin{aligned}
Y_{t, \sigma}^{i}\left(\theta_{1}, \cdots, \theta_{i}\right) & =B_{\sigma}^{i}\left(\theta_{1}, \cdots, \theta_{i}\right)+\int_{t}^{T} f_{\sigma}^{i}\left(s, Y_{s, \sigma}^{i}\left(\theta_{1}, \cdots, \theta_{i}\right), Z_{s, \sigma}^{i}\left(\theta_{1}, \cdots, \theta_{i}\right), Y_{s, \sigma}^{i+1}\left(\theta_{1}, \cdots, \theta_{i}, s\right)\right. \\
& \left.-Y_{s, \sigma}^{i}\left(\theta_{1}, \cdots, \theta_{i}\right), \theta_{1}, \cdots, \theta_{i}\right) d s-\int_{t}^{T} Z_{s, \sigma}^{i}\left(\theta_{1}, \cdots, \theta_{i}\right) d W_{s} \\
Y_{t, \sigma}^{0} & =B^{0}+\int_{t}^{T} f^{0}\left(s, Y_{s, \sigma}^{0}, Z_{s, \sigma}^{0}, Y_{s, \sigma}^{1}(s)-Y_{s, \sigma}^{0}\right) d s-\int_{t}^{T} Z_{s, \sigma}^{0} d W_{s} \\
Y_{t, \sigma}^{n}\left(\theta_{1}, \cdots, \theta_{i}\right) & =B^{n}(\tau(1, n)) .
\end{aligned}
$$

Under these assumptions, the solution of (A1) is

$$
\begin{aligned}
& Y_{t}= \sum_{\sigma \in \mathfrak{S}_{n}} \mathbf{1}_{\left\{\tau_{\sigma(1)} \leq \tau_{\sigma(2)} \cdots \leq \tau_{\sigma(n)}\right\}}\left[Y_{t, \sigma}^{0} \mathbf{1}_{\left\{t<\tau_{\sigma(1)}\right\}}+\sum_{i=1}^{n-1} \mathbf{1}_{\left\{\tau_{\sigma(i)} \leq t<\tau_{\sigma(i+1)}\right\}} Y_{t, \sigma}^{i}(\tau(1, i))\right. \\
&\left.\quad+\mathbf{1}_{\left\{\tau_{\sigma(n)} \leq t\right\}} B^{n}(\tau(1, n))\right], \\
& Z_{t}= \sum_{\sigma \in \mathfrak{S}_{n}} \mathbf{1}_{\left\{\tau_{\sigma(1)} \leq \tau_{\sigma(2)} \cdots \leq \tau_{\sigma(n)}\right\}}\left[Z_{t, \sigma}^{0} \mathbf{1}_{\left\{t \leq \tau_{\sigma(1)}\right\}}+\sum_{i=1}^{n-1} \mathbf{1}_{\left\{\tau_{\sigma(i)}<t \leq \tau_{\sigma(i+1)}\right\}} Z_{t, \sigma}^{i}(\tau(1, i))\right], \\
& U_{t}^{i}=\sum_{\sigma \in \mathfrak{S}_{n}} \mathbf{1}_{\left\{\tau_{\sigma(1)} \leq \tau_{\sigma(2)} \cdots \leq \tau_{\sigma(n)}\right\}} \sum_{j=1}^{n} \mathbf{1}_{\{\sigma(j)=i\}} \mathbf{1}_{\left\{\tau_{\sigma(j-1)}<t \leq \tau_{\sigma(j)}\right\}}\left(Y_{t, \sigma}^{j}(\tau(1, j))-Y_{t, \sigma}^{j-1}(\tau(1, j-1))\right),
\end{aligned}
$$

where by convention $\tau_{\sigma(0)}=0$ and $Y_{t, \sigma}^{0}(\tau(1,0))=Y_{t, \sigma}^{0}$.

In the above proposition, the function $f$ may be written a :

$$
\begin{aligned}
f(t, z, u) & =\mathbf{1}_{\left\{t<\underline{\tau}^{1 n}\right\}}\left[\sum_{i=1}^{n}\left(1-H_{t}^{i}\right) \frac{e^{\alpha U_{t}^{i}}-1}{\alpha} \lambda_{t}^{i}-\frac{z\left(\mu_{t}-r\right)}{\sigma_{t}}-\frac{1}{2 \alpha} \frac{\left(\mu_{t}-r\right)^{2}}{\sigma_{t}^{2}}\right] \\
& +\sum_{\sigma \in \Sigma_{n}} \sum_{i=1}^{n-1} \mathbf{1}_{\left\{\bar{\tau}_{\sigma}^{i} \leq t<\underline{\tau}_{\sigma}^{i+1, n}\right\}}\left[\sum_{j=i+1}^{n}\left(1-H_{t}^{\sigma(j)}\right) \frac{e^{\alpha U_{t}^{\sigma(j)}}-1}{\alpha} \lambda_{t}^{\sigma(j)}-\frac{z\left(\mu_{t}-r\right)}{\sigma_{t}}-\frac{1}{2 \alpha} \frac{\left(\mu_{t}-r\right)^{2}}{\sigma_{t}^{2}}\right], \\
& +\mathbf{1}_{\left\{\bar{\tau}^{1 n} \leq t\right\}}\left[-\frac{z\left(\mu_{t}-r\right)}{\sigma_{t}}-\frac{1}{2 \alpha} \frac{\left(\mu_{t}-r\right)^{2}}{\sigma_{t}^{2}}\right] .
\end{aligned}
$$

A.2 Proof of Proposition 3. The proof follows similar arguments as in Kharroubi and Lim (2014) and Kharroubi et al. (2013). The major difference is that we distinguish two cases corresponding to the sets $\left\{\tau_{1}<\tau_{2}\right\}$ and $\left\{\tau_{2}<\tau_{1}\right\}$.

Let us first suppose that $\tau_{1}<\tau_{2}$. The main idea of the proof is to make an induction on the number $k$ of jumps in $(t, T]$. The proof falls then naturally into two parts as follows.

- Suppose that $k=0$. Then, we distinguish three cases. 
- Case 1: on $\left\{0 \leq \tau_{1}<\tau_{2} \leq t<T\right\}$. Then $Y_{t}=B^{3}\left(\tau_{1}, \tau_{2}\right)$ therefore

$$
Y_{t}=B^{3}+\int_{t \wedge\left(\tau_{1} \vee \tau_{2}\right)}^{T \wedge\left(\tau_{1} \vee \tau_{2}\right)} f\left(s, Z_{s}, U_{s}^{1}, U_{s}^{2}\right) d s-\int_{t \wedge\left(\tau_{1} \vee \tau_{2}\right)}^{T \wedge\left(\tau_{1} \vee \tau_{2}\right)} Z_{s} d W_{s}-\int_{t \wedge\left(\tau_{1} \vee \tau_{2}\right)}^{T \wedge\left(\tau_{1} \vee \tau_{2}\right)}\left(U_{s}^{1} d H_{s}^{1}+U_{s}^{2} d H_{s}^{2}\right) .
$$

- Case 2: on $\left\{\tau_{1} \leq t<T<\tau_{2}\right\}$, hence $Y_{t}=Y_{t}^{1}$ and since there is no jumps between $t$ and $T$, $Y_{s}=Y_{s}^{1}, Z_{s}=Z_{s}^{1}, U_{s}^{1}=0, U_{s}^{2}=B^{3}(., s)-Y_{s}^{1}$ for all $s \in(t, T]$ and $\int_{t}^{T}\left(U_{s}^{1} d H_{s}^{1}+U_{s}^{2} d H_{s}^{2}\right)=$ 0 . Then we conclude that on $\left\{\tau_{1} \leq t<T<\tau_{2}\right\}$

$$
Y_{t}=B+\int_{t \wedge\left(\tau_{1} \vee \tau_{2}\right)}^{T \wedge\left(\tau_{1} \vee \tau_{2}\right)} f\left(s, Z_{s}, U_{s}^{1}, U_{s}^{2}\right) d s-\int_{t \wedge\left(\tau_{1} \vee \tau_{2}\right)}^{T \wedge\left(\tau_{1} \vee \tau_{2}\right)} Z_{s} d W_{s}-\int_{t \wedge\left(\tau_{1} \vee \tau_{2}\right)}^{T \wedge\left(\tau_{1} \vee \tau_{2}\right)}\left(U_{s}^{1} d H_{s}^{1}+U_{s}^{2} d H_{s}^{2}\right)
$$

- Case 3: on $\left\{t<T \leq \tau_{1}<\tau_{2}\right\}$, hence $Y_{t}=Y_{t}^{0,1}$ and since there is no jumps between $t$ and $T, Y_{s}=Y_{s}^{0,1}, Z_{s}=Z_{s}^{0,1}, U_{s}^{1}=Y_{s}^{1}(s)-Y_{s}^{0,1}, U_{s}^{2}=0$ for all $s \in(t, T]$ and $\int_{t}^{T}\left(U_{s}^{1} d H_{s}^{1}+\right.$ $\left.U_{s}^{2} d H_{s}^{2}\right)=0$. Then we conclude that on $\left\{t<T \leq \tau_{1}<\tau_{2}\right\}$

$$
Y_{t}=B+\int_{t \wedge\left(\tau_{1} \vee \tau_{2}\right)}^{T \wedge\left(\tau_{1} \vee \tau_{2}\right)} f\left(s, Z_{s}, U_{s}^{1}, U_{s}^{2}\right) d s-\int_{t \wedge\left(\tau_{1} \vee \tau_{2}\right)}^{T \wedge\left(\tau_{1} \vee \tau_{2}\right)} Z_{s} d W_{s}-\int_{t \wedge\left(\tau_{1} \vee \tau_{2}\right)}^{T \wedge\left(\tau_{1} \vee \tau_{2}\right)}\left(U_{s}^{1} d H_{s}^{1}+U_{s}^{2} d H_{s}^{2}\right)
$$

- Suppose that there is $k=1$ jump.

- Case 1: On $\left\{t \leq \tau_{1}<T<\tau_{2}\right\}$, hence $Y_{t}=Y_{t}^{0,1}$. Then $Y_{s}=Y_{s}^{0,1}, Z_{s}=Z_{s}^{0,1}, U_{s}^{1}=$ $Y_{s}^{1}(s)-Y_{s}^{0,1}, U_{s}^{2}=0$ for all $s \in\left(t, \tau_{1}\right]$

$$
\begin{aligned}
Y_{t} & =Y_{t}^{0,1}=Y_{\tau_{1}}^{0,1}+\int_{t}^{\tau_{1}} f\left(s, Z_{s}, U_{s}^{1}, U_{s}^{2}\right) d s-\int_{t}^{\tau_{1}} Z_{s}^{0,1} d W_{s}, \\
& =Y_{\tau_{1}}^{1}+\int_{t}^{\tau_{1}} f\left(s, Z_{s}, U_{s}^{1}, U_{s}^{2}\right) d s-\int_{t}^{T} Z_{s}^{0,1} 1_{s \leq \tau_{1}} d W_{s}-\int_{t}^{\tau_{1}}\left(U_{s}^{1} d H_{s}^{1}+U_{s}^{2} d H_{s}^{2}\right) .
\end{aligned}
$$

Then using similar arguments as Kharroubi and Lim (2014), we have

$$
Y_{t}=Y_{\tau_{1}}^{1}+\int_{t}^{\tau_{1}} f\left(s, Z_{s}, U_{s}^{1}, U_{s}^{2}\right) d s-\int_{t}^{T} Z_{s} 1_{s \leq \tau_{1}} d W_{s}-\int_{t}^{\tau_{1}}\left(U_{s}^{1} d H_{s}^{1}+U_{s}^{2} d H_{s}^{2}\right) .
$$

- Case 2: On $\left\{t \leq \tau_{1}<T<\tau_{2}\right\}$. As there is no jump on $\left(\tau_{1}, T\right]$, the previous result implies that on $\left\{t \leq \tau_{1}<T<\tau_{2}\right\}$,

$$
Y_{r}=B+\int_{r}^{T} f\left(s, Z_{s}, U_{s}^{1}, U_{s}^{2}\right) d s-\int_{r}^{T} Z_{s} d W_{s}-\int_{r}^{T}\left(U_{s}^{1} d H_{s}^{1}+U_{s}^{2} d H_{s}^{2}\right) .
$$

So we obtain that

$$
Y_{\tau_{1}}=Y_{\tau_{1}}^{1}=B+\int_{r}^{T} f\left(s, Z_{s}, U_{s}^{1}, U_{s}^{2}\right) d s-\int_{r}^{T} Z_{s} d W_{s}-\int_{r}^{T}\left(U_{s}^{1} d H_{s}^{1}+U_{s}^{2} d H_{s}^{2}\right) .
$$


- Case 3: Finally on $\left\{t \leq \tau_{1}<T<\tau_{2}\right\}$, we have

$$
Y_{t}=B+\int_{t \wedge\left(\tau_{1} \vee \tau_{2}\right)}^{T \wedge\left(\tau_{1} \vee \tau_{2}\right)} f\left(s, Z_{s}, U_{s}^{1}, U_{s}^{2}\right) d s-\int_{t \wedge\left(\tau_{1} \vee \tau_{2}\right)}^{T \wedge\left(\tau_{1} \vee \tau_{2}\right)} Z_{s} d W_{s}-\int_{t \wedge\left(\tau_{1} \vee \tau_{2}\right)}^{T \wedge\left(\tau_{1} \vee \tau_{2}\right)}\left(U_{s}^{1} d H_{s}^{1}+U_{s}^{2} d H_{s}^{2}\right)
$$

The other cases follow from the same arguments. 